\section{The Hsp70 chaperone system: distinct roles in erythrocyte formation and maintenance}

\author{
Yasith Mathangasinghe, ${ }^{1}$ Bruno Fauvet, ${ }^{2}$ Stephen M. Jane, ${ }^{3,4}$ Pierre \\ Goloubinoff $^{2}$ and Nadinath B. Nillegoda ${ }^{1}$
}

${ }^{1}$ Australian Regenerative Medicine Institute, Monash University, Clayton, Victoria, Australia; ${ }^{2}$ Department of Plant Molecular Biology, Lausanne University, Lausanne, Switzerland; ${ }^{3}$ Central Clinical School, Monash University, Prahran, Victoria, Australia and ${ }^{4}$ Department of Hematology, Alfred Hospital, Monash University, Prahran, Victoria, Australia

\section{ABSTRACT}

E rythropoiesis is a tightly regulated cell differentiation process in which specialized oxygen- and carbon dioxide-carrying red blood cells are generated in vertebrates. Extensive reorganization and depletion of the erythroblast proteome leading to the deterioration of general cellular protein quality control pathways and rapid hemoglobin biogenesis rates could generate misfolded/aggregated proteins and trigger proteotoxic stresses during erythropoiesis. Such cytotoxic conditions could prevent proper cell differentiation resulting in premature apoptosis of erythroblasts (ineffective erythropoiesis). The heat shock protein 70 (Hsp70) molecular chaperone system supports a plethora of functions that help maintain cellular protein homeostasis (proteostasis) and promote red blood cell differentiation and survival. Recent findings show that abnormalities in the expression, localization and function of the members of this chaperone system are linked to ineffective erythropoiesis in multiple hematological diseases in humans. In this review, we present latest advances in our understanding of the distinct functions of this chaperone system in differentiating erythroblasts and terminally differentiated mature erythrocytes. We present new insights into the protein repair-only function(s) of the Hsp70 system, perhaps to minimize protein degradation in mature erythrocytes to warrant their optimal function and survival in the vasculature under healthy conditions. The work also discusses the modulatory roles of this chaperone system in a wide range of hematological diseases and the therapeutic gain of targeting Hsp70.

\section{Introduction}

Cells are highly vulnerable to proteotoxic stresses during widespread remodeling of proteomes that typically accompany cell differentiation. Under such challenging conditions, molecular chaperones that constitute an essential part of cellular protein quality control (POC) pathways, help maintain proteostasis by decreasing protein misfolding and aggregation, and promote cell viability. ${ }^{1,2}$ In response to cell differentiation, considerable rearrangements in cellular chaperomes have been detected, ${ }^{2,3}$ but the functional consequences of such changes largely remain enigmatic. In particular, the heat shock protein 70 (Hsp70) chaperone system is maintained at high levels during red blood cell differentiation. ${ }^{3.5}$ Emerging data demonstrate that Hsp70 machineries have distinct functions ranging from modulating cell signaling to PQC activities at different stages of erythropoiesis. These multifaceted roles of the Hsp70 chaperone include maintaining erythroid progenitors, assessing fitness of progenitors prior to initiating lineage specific terminal cell differentiation, supporting hemoglobin $(\mathrm{Hb})$ biogenesis, counteracting proteotoxicities and preventing premature apoptosis of differentiating erythroblasts, and promoting viability of terminally differentiated erythrocytes via protein repair. Hence, the dysfunction of this chaperone system is invariably associated with ineffective erythropoiesis, which leads to chronic anemia in several hematological diseases in humans.
Ferrata Storti Foundation

Haematologica 2021

Volume 106(6):1519-1534

\section{Correspondence:}

NADINATH B. NILLEGODA

nadinath.nillegoda@monash.edu

Received: July 29, 2019.

Accepted: September 25, 2020.

Pre-published: April 8, 2021.

https://doi.org/10.3324/haematol.2019.233056

(C)2021 Ferrata Storti Foundation

Material published in Haematologica is covered by copyright. All rights are reserved to the Ferrata Storti Foundation. Use of published material is allowed under the following terms and conditions:

https://creativecommons.org/licenses/by-nc/4.0/legalcode. Copies of published material are allowed for personal or internal use. Sharing published material for non-commercial purposes is subject to the following conditions:

https://creativecommons.org/licenses/by-nc/4.0/legalcode, sect. 3. Reproducing and sharing published material for commercial purposes is not allowed without permission in writing from the publisher. 


\section{Formation of red blood cells}

Erythropoiesis is a vital process throughout vertebrate life, which helps maintain adequate tissue oxygenation under physiological and nonphysiological states (e.g., hypoxia, hemorrhage or other anemic conditions). This cell differentiation event leads to the generation of highly specialized erythrocytes that function as dedicated oxygen and carbon dioxide transporting cells across the body. Erythrocytes have a finite lifespan (approximately 120 days in humans) in the circulatory system before they are recycled mainly in the spleen by macrophages. ${ }^{6}$ These cells, therefore, must be continuously and rapidly replaced in vertebrates. About two million new erythrocytes per second are generated in adult humans ${ }^{7}$ via proliferation and differentiation of a self-renewing population of pluripotent hematopoietic stem cells (HSC) located in the yolk sac, liver, spleen (antenatal) or bone marrow (postnatal) that give rise to early erythroid progenitors. ${ }^{6}$ During erythropoiesis, these progenitors undergo a red cell lineage specific terminal differentiation program to generate mature erythrocytes.

Erythrocyte production is tightly regulated by a set of hormones. For example, glucocorticoids regulate both the proliferation and differentiation of early erythroid progenitors known as early burst forming unit-erythroid (BFU-E) cells. ${ }^{8}$ The proliferation and differentiation of subsequent erythroid progenitors including late stage BFU-E and colony forming unit-erythroid (CFU-E) cells occur after the stimulation by erythropoietin (EPO), a glycoprotein cytokine secreted by the kidneys. ${ }^{9}$ EPO stimulation is vital for the induction of GATA-binding factor 1 (GATA-1) transcription factor, the master regulator of erythropoiesis. GATA-1 together with the transcription factor STAT5, promote further erythroblast proliferation ${ }^{10}$ and turn on the gene activation and repression program, which drives the multistep terminal differentiation process of these cells. ${ }^{11}$ In mammals, erythropoiesis can be resolved into six morphologically distinct cell stages that result from a series of mitotic cell divisions (Figure 1A). These stages include: (i) proerythroblast, (ii) basophilic erythroblast, (iii) polychromatophilic erythroblast, (IV) orthochromatophilic erythroblast, (V) reticulocyte, and (VI) mature erythrocyte. During terminal differentiation, the erythroblasts decrease in cell size, condense chromatin, reorganize and reduce cellular proteome and membranes, and eliminate organelles, thereby making space for the rapidly increasing levels of $\mathrm{Hb}$, the oxygen-trafficking protein complex. The most striking morphological change occurs in orthochromatophilic erythroblasts that eject nuclei to form reticulocytes in the bone marrow (Figure 1A). These reticulocytes loose ribosomes and the bulk of RNA molecules, and develop into $\mathrm{Hb}$ packed mature erythrocytes that have a characteristic biconcave disk-like shape with a flattened center.

The mature erythrocytes contain a remarkably high concentration of $\mathrm{Hb}$ molecules (approximately 29.5 $\mathrm{pg} / \mathrm{cell}){ }^{12}$ which represents approximately $98 \%$ of the proteome. ${ }^{13} \mathrm{Hb}$ is a tetrameric globular protein made up of four globin protein subunits/chains, each containing a heme group, which reversibly binds to oxygen and carbon dioxide. The adult human hemoglobin $(\mathrm{HbA})$ is made from two 141 amino acid-long $\alpha$-globin chains and two 146 amino acid-long $\beta$-globin chains $\left(\alpha_{2} \beta_{2}\right)$ (Figure $1 B)$. Under physiological conditions, $\mathrm{HbA}$ makes up
$>97 \%$ of the $\mathrm{Hb}$ constituent in adult humans. The remaining $\mathrm{Hb}$ contains fetal $\mathrm{Hb}\left(\mathrm{HbF} ; \alpha_{2} \gamma_{2}\right)$ and $\mathrm{HbA} 2$ $\left(\alpha_{2} \delta_{2}\right)$, two isotypes generated by switching the $\beta$ globin chain with either $\gamma$ or $\delta$ globin chains. ${ }^{6}$

\section{Proteotoxic stresses associated with erythropoiesis}

Proteostasis is maintained by balancing the cellular pathways that facilitate protein synthesis, folding, assembly, trafficking, and degradation under varying environmental and metabolic conditions. ${ }^{1}$ Even under normal growth conditions, cells experience a continuous influx of misfolded proteins generated from various protein biogenesis mistakes such as errors in transcription, translation and folding. Additionally, most proteins are at risk of misfolding due to the marginal stability of their native conformations. ${ }^{14}$ Cells have evolved a set of intricate PQC pathways comprising of molecular chaperones and protein degradation systems that operate constantly to decrease the levels of misfolded proteins that otherwise would easily form aggregates in crowded cellular environments. Protein aggregates typically show poor solubility in aqueous cellular environments, have no physiological function per se and could instead elicit cytotoxicity. ${ }^{1}$ By untangling and unfolding such aberrant protein species, the ATP fueled chaperone machineries are able to "repair" and rescue proteins, which leads to a considerable reduction in the risk of proteotoxicities in cells. Protein degradation pathways also represent an important line of defense by clearing misfolded proteins and preventing their accumulation. ${ }^{1}$ However, in aging and/or stressed cells, such defense mechanisms could become overwhelmed leading to the buildup of potentially toxic protein aggregate species. Many disease-linked mutant proteins also form such aggregates that are refractory to PQC systems, including degradation pathways. ${ }^{15}$

Due to various protein biosynthetic errors and considerable attenuation of basic PQC pathways, erythroid maturation is highly exposed to protein misfolding/aggregation. The first PQC challenge during erythropoiesis involves the folding and assembly of the $\alpha$-globin chains that show a high degree of instability. In the absence of the partnering $\beta$-globin, the free $\alpha$-chains with heme/iron could readily misfold to form protein aggregate deposits called Heinz bodies (for a review, see Voon et al. ${ }^{16}$ ). These highly toxic protein aggregates, when accumulated, could trigger the generation of reactive oxygen species (ROS) $)^{17}$ that damage cellular proteins, nucleic acids and lipids and induce oxidative stress in erythroblasts leading to premature cell death (Figure 1B). ${ }^{18}$ This is circumvented to a certain degree with the assistance of a dedicated chaperone named alpha hemoglobin stabilizing protein (AHSP). AHSP mimics the $\alpha$-helix-loop- $\alpha$-helix motif of $\beta$-globin and assists in the folding of $\alpha$-chains in a "template" directed manner ${ }^{19,20}$ (for a review, see Weiss et al.) ${ }^{21}$. Apart from the $\alpha$-chain instability, the generally high synthesis rates of globin proteins $(300 \mathrm{mg}$ of $\mathrm{Hb}$ per hour in healthy adult humans ${ }^{22}$ ) during this atypical state could also proportionally increase the level of intrinsic errors in folding and assembly ${ }^{23}$ of $\mathrm{Hb}$. In particular, heme/iron imbalances could result in globin misfolding, which could induce severe oxidative stress in erythroblasts. A tightly regulated supply of iron to support the production of heme is 
required for efficient $\mathrm{Hb}$ biogenesis. Heme synthesis is mediated by conjugating iron and protoporphyrin in a series of enzymatic reactions occurring in mitochondria and cytosol. ${ }^{24}$ The hydroxyl radicals produced by elevated levels of free heme/iron undergoing the Fenton reaction in erythroblast cytosol could damage and induce the aggregation of both $\mathrm{Hb}$ and other critical biomolecules. ${ }^{25}$
The next PQC challenge in red blood cell maturation occurs from the reduction/disruption of crucial PQC pathways that typically protect cells against protein aggregation. In order to make space for the increasing levels of $\mathrm{Hb}$, the proteome of the terminally differentiating erythroblasts is rapidly reduced to $2-5 \%$ via bulk degradation of many cellular proteins and organelles ${ }^{3}$ by the

A

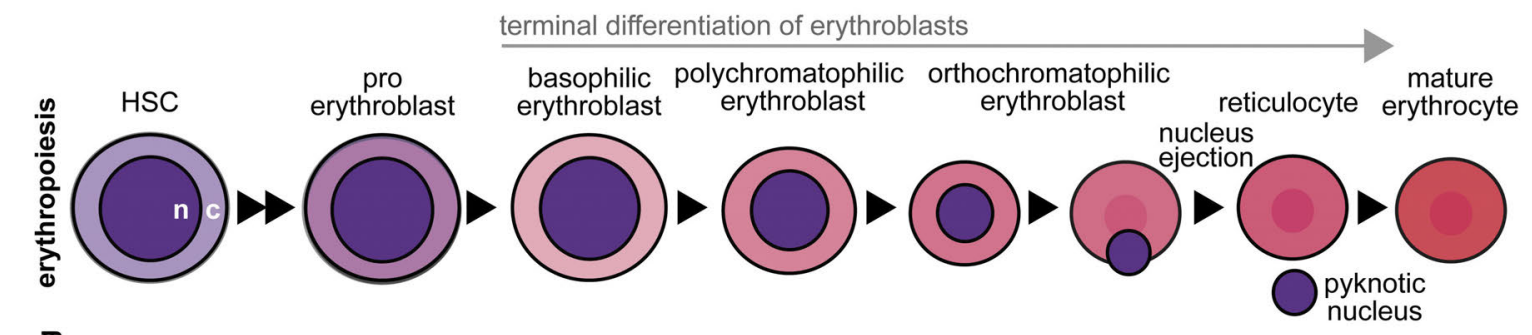

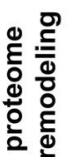

hemoglobin level non-hemoglobin proteome level

B

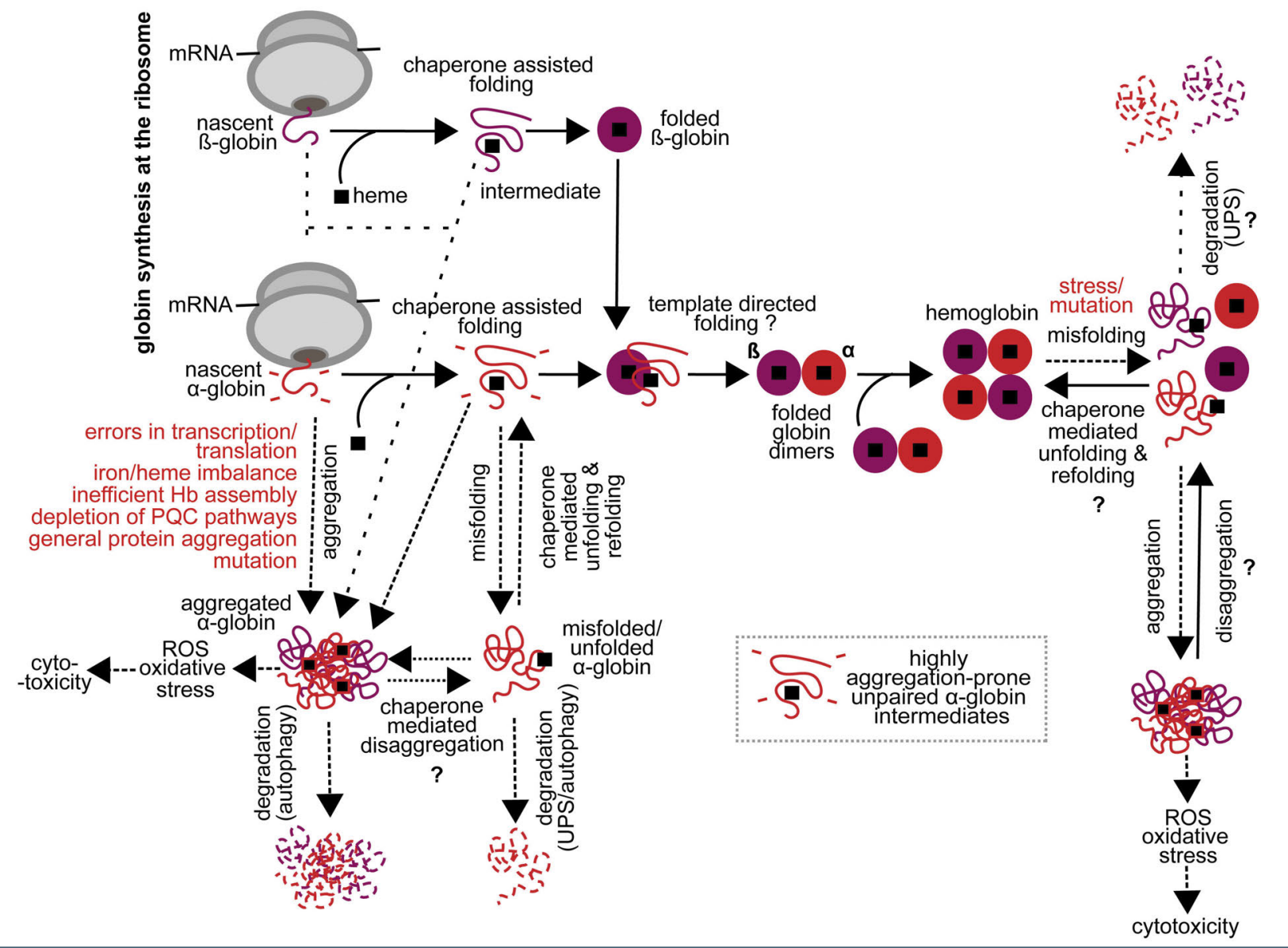

Figure 1. Biogenesis of hemoglobin during erythropoiesis. (A) A simplified schematic diagram showing the key cell stages of erythropoiesis. Hematopoietic stem cells (HSC) differentiate into a common myeloid progenitor, which further transition into a committed erythroid lineage. The proerythroblast is the earliest morphologically identifiable erythroid precursor cell in the bone marrow. Erythropoietin (EPO) signaling initiates terminal differentiation of erythroblasts to generate mature erythrocytes. During terminal differentiation, cells reduce in size and undergo major changes including chromatin condensation, proteome remodeling and ultimately the elimination of cellular organelles to provide room for hemoglobin $(\mathrm{Hb})$. $\mathrm{Hb}$ expression levels in differentiating cells are indicated by intensity of the red color. (B) Chaperone assisted folding and assembly of $\mathrm{Hb}$. The folding of nascent globin chains is assisted by chaperones such as the $\alpha$-Hb-stabilizing protein (AHSP), heat shock protein 70 (Hsp70) and heat shock protein 90 (Hsp90). Errors in Hb subunit synthesis and assembly, iron/heme imbalances, deficiencies in protein quality control activities and exposure to reactive oxygen species (ROS), however, could trigger the misfolding and aggregation of globin proteins. In particular, misfolded and unassembled $\alpha$-globin chains are highly prone to form cytotoxic aggregates leading to ineffective erythropoiesis. c: cytosol; n: nucleus; UPS: ubiquitin proteasome system. 
ubiquitin proteasome system (UPS) and the autophagy pathway. ${ }^{26,27}$ This drastic proteome remodeling expectedly depletes components of chaperone and proteolytic machineries and decreases the ability of these cells to induce global PQC pathways that help buffer against proteotoxic stresses..$^{28}$ For example, unlike other cell types, reticulocytes show inability to fully recover critical cellular functions such as protein synthesis after heat shock. ${ }^{4,29}$ On the whole, terminally differentiating erythroblasts seem particularly vulnerable to stresses associated with protein misfolding and aggregation.

\section{The Hsp70 chaperone system}

Hsp70 forms one of the most abundant and highly conserved molecular chaperone systems critical for maintaining cellular proteostasis. This highly versatile chaperone system supports a plethora of housekeeping and stressrelated cellular repair processes that protect cells against proteotoxic stresses (for a review, see Rosenzweig et al..$^{30}$ ). The key housekeeping activities include facilitating folding of newly synthesized proteins, transport of polypeptides across cellular membranes, assembly/disassembly of protein complexes and regulation of protein activity. In stressed cells, the Hsp70 chaperone system functions to prevent aggregation of aberrant proteins, refold misfolded proteins, solubilize aggregated proteins, and cooperate with cellular degradation machineries to clear terminally damaged proteins (for a review, see Rosenzweig et al..$^{30}$ ).

The chaperoning functions of Hsp70 are tightly regulated via the cooperation of dedicated cochaperones from the J-domain protein (JDP) family and nucleotide exchange factors (NEF) that fine-tune Hsp70's ATPdependent allosteric control of substrate binding and release (Figure 2A). JDP form the largest and the most diverse family of cochaperones in humans (over $42 \mathrm{mem}$ bers) and provide specificity to the Hsp70 family (13 homologs in humans) by selecting substrates. ${ }^{30,31}$ Concomitant interaction of Hsp70 with a JDP and substrate boosts ATP hydrolysis in Hsp70. This dual trigger allows Hsp70 to efficiently trap and unfold substrates (Figure 2A). ${ }^{32}$ The timely release of substrates is mediated by nucleotide exchange factors that release $\mathrm{ADP}$ and allow subsequent rebinding of ATP, thus resetting the Hsp70 chaperone to its open, low substrate affinity state to receive a new client. ${ }^{30,33}$

Hsp70 generally shows a high affinity towards misfolded and aggregated substrates, and a low affinity for native proteins, which may, thus be considered as the products of such polypeptide unfolding enzymes. ${ }^{34}$ As earlier stated, the energy from ATP hydrolysis drives the iterative protein unfolding cycles of $\mathrm{Hsp} 70$ that allow the refolding of misfolded proteins (Figure 2A). Hsp70 and other protein folding chaperone systems (e.g., Hsp60 and Hsp90) promote the buildup and maintenance of relatively high levels of native protein conformers under non-equilibrium stress conditions where without chaperones or ATP, the denatured protein conformers would readily seek equilibrium and turn into stable inactive misfolded species. ${ }^{35}$ Conceptually, this aligns with Erwin Schrödinger's view, which states that living matter evades the decay to equilibrium. ${ }^{36}$ The term "evades" implies that living cells must constantly consume energy in order to avoid spontaneous entropy-driven decomposition of their macromolecules (e.g., proteins), leading to cell death. This is possible because the biosphere is not a closed system: the energy from the sun is harnessed by photosynthesis to produce ATP for all organisms to fuel their repair (and replace) mechanisms. The chaperone-based protein repair mechanisms constantly counteract the natural entropic tendency of proteins to misfold and further decay by hydrolysis and oxidation into simpler molecules. In other words, when acting as ATP-fueled iterative unfolding nanomachines, chaperones such as Hsp70 can correct or "repair" structurally damaged proteins as they are formed under stressful non-equilibrium conditions. ${ }^{37}$ Erythropoiesis is a prime example of how Hsp70's protein repair and regulatory functions are fully deployed to support cell differentiation and viability.

\section{Multifaceted roles of the Hsp70 chaperone system in erythropoiesis}

During red blood cell generation, the Hsp70 chaperone system functions in a number of regulatory and PQC activities. By changing its cochaperones, the Hsp70 chaperone could target different clientele and switch between functions, which allows this highly versatile chaperone system to rapidly respond to different cell growth and differentiation conditions. ${ }^{38,39}$ The main roles of Hsp70 during erythropoiesis include: (i) aiding in maintaining erythroid progenitors (ii) assessing fitness of progenitors prior to initiating lineage specific terminal cell differentiation (iii) supporting $\mathrm{Hb}$ biogenesis (iv) counteracting proteotoxicities and preventing premature apoptosis of differentiating erythroblasts and (v) conceivably promoting viability of differentiated (mature) erythrocytes via protein repair.

\section{Hsp70 regulates dormancy and cell cycle quiescence of erythroid precursor cells}

Continuous proliferation and differentiation of hematopoietic stem cells into committed erythroid progenitor cells $s^{6}$ is required for maintaining healthy levels of mature erythrocytes in the peripheral vasculature. The cyclin dependent cell cycle entry from G1 to S phase during proliferation of hematopoietic stem cells is modulated largely by the opposing actions of cyclin dependent kinases (CDK) and cyclin-dependent kinase inhibitors (CDKi). ${ }^{40}$ In order to terminate the dormancy of hematopoietic stem cells and initiate cell cycle entry, cyclin D1, the regulatory subunit of CDK4 and CDK6, has to translocate from the cytosol to the nucleus. ${ }^{41,42}$ This key step in HSC proliferation is mediated by the constitutively expressed heat shock cognate protein 70 (Hsc70/HSPA8), which binds to cyclin D1 and shuttles it across the nuclear membrane (Figure 3) ${ }^{41}$ Here, HSPA8 appears to recognize a peptide segment in an unstructured (likely a looped or terminal) region exposed on the surface of folded cyclin D1. Similar types of interactions between Hsp70 and native proteins leading to regulatory activities have been demonstrated with clathrin triskelions, immunoglobulin heavy chain, Escherichia coli heat shock transcription factor $\sigma 32$ and plasmid replication protein RepE..$^{43-45}$ The cyclin D1-HSPA8 complex is retained in the cytosol by forming additional interaction(s) with p57 $57^{\mathrm{KI} 2}$ and $\mathrm{p} 27^{\mathrm{KIP} 1}$, two critical CDKi that are known to prevent the cell cycle progression of 
hematopoietic stem cells (Figure 3). ${ }^{41}$ It is tempting to speculate that through complexing, the CDKi mask the nuclear localization related signal of HSPA8 $8^{41,42}$ until the CDKi are degraded via stem cell factor signaling coupled to the initiation of erythropoiesis. ERK signaling, which plays a modulatory role in erythropoiesis ${ }^{46}$ also plays a role in the nuclear shuttling of $\mathrm{Hsp} 70,{ }^{47}$ but little is known about how these signals are integrated, if at all. The rodent mammalian relative of DnaJ (MRJ), an ortholog of human JDP DNAJB6, was identified from recent stem cell work $^{48}$ to play a role in promoting cell quiescence by binding to a cyclin D1 inhibitor. ${ }^{49}$ The same JDP was implicated in playing a role in stem cell self-renewal. ${ }^{50}$ Whether MRJ/DNAJB6 or another JDP directs the selection of clients in the CDKi-HSPA8-cyclin D1-mediated HSC proliferation pathway remains to be determined. The terminal differentiation of erythroblasts also requires cell cycle regulating cyclins. Cyclins A2 and D3 are required to control cytokinesis, erythrocyte size and number. ${ }^{51,52}$ Here too, the Hsp70 system facilitates the
A

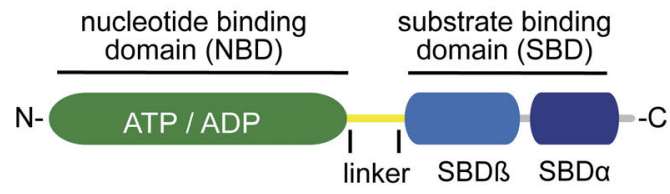

low affinity (ATP) state

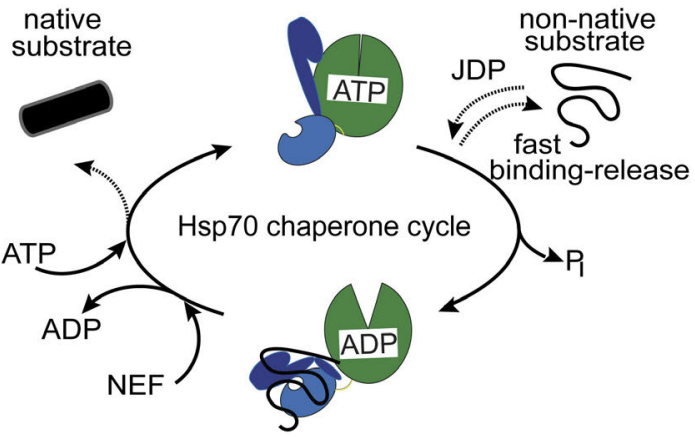

high affinity (ADP) state
B

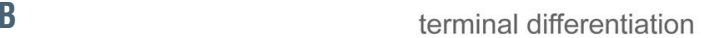

ifferentiation

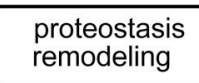

remodeling

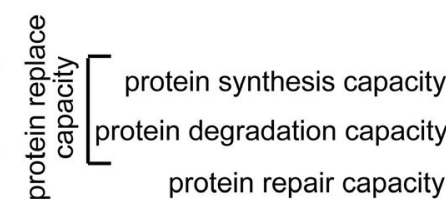

protein repair capacity

\section{(1)}

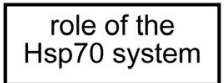

$$
\begin{array}{cc}
\begin{array}{c}
\text { maintenance } \\
\text { of progenitors }
\end{array} & \begin{array}{c}
\text { fitness } \\
\text { checkpoints }
\end{array} \\
\text { PQC } & \text { PQC } \\
\begin{array}{c}
\text { (protein biogenesis, } \\
\text { repair and/or } \\
\text { replace) }
\end{array} & \text { signalionaling } \\
\text { regulation/signaling } &
\end{array}
$$

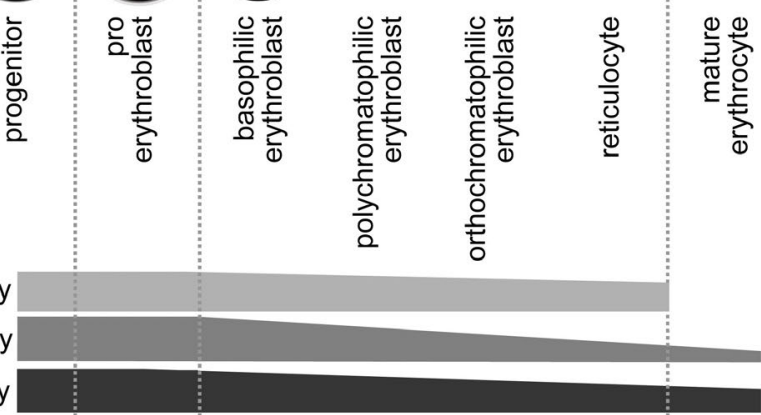

(3) cell survival/ differentiation

regulation/signaling anti-apoptotic activity
(4)

cell survival restricted PQC (protein repair-only)

Figure 2. Roles of the Hsp70 chaperone system in differentiating erythroblasts and mature erythrocytes. (A) Domain organization of the heat shock protein 70 (Hsp70) chaperone (top); the Hsp70 chaperoning cycle (bottom). Substrate binding is dictated in Hsp70 by the allosteric coupling of ATP binding and hydrolysis at the N-terminal nucleotide binding domain (NBD), which results in conformational changes at the substrate binding domain (SBD). ${ }^{147}$ The conformational cycle linked to substrate capture is defined by ATP hydrolysis driven large scale movements in the $\alpha$-helical-lid domain (SBD $\alpha$ ) that closes over the $\beta$-sandwich substrate binding subdomain (SBD $\beta$ ) in the ADP state, resulting in low substrate off-rates (i.e., high affinity towards bound substrates)..$^{30} \mathrm{~J}$-domain proteins (JDP) select substrates for Hsp70. Concomitant interactions of the Hsp70 (in ATP state) with JDP and substrate result in increased stimulation of ATP hydrolysis trapping the substrate in Hsp70. Subsequently, nucleotide exchange factors (NEF) induce ADP dissociation from Hsp70 allowing ATP rebinding, which triggers substrate release to complete Hsp70 cycle. Substrate unfolding and refolding is facilitated by multiple cycles of substrate binding and release. Hsp70 recognizes a highly degenerative and frequently occurring peptide motif enriched with five hydrophobic amino acids, flanked by preferentially positively charged amino acids (statistically occurring in every $30-40$ residues in polypeptide chains). ${ }^{148}$ Such hydrophobic motifs are typically buried inside a natively folded protein, but become exposed in unfolded or misfolded conformers, which allow the Hsp70 machinery to discriminate between natively folded and unfolded/misfolded/aggregated substrates. ${ }^{30}$ (B) Expression profiles of selected chaperone systems in different stages of erythropoiesis from quantitative proteomics data. ${ }^{3,5}$ The black dotted line represents the median relative abundance of non-hemoglobin $(\mathrm{Hb})$ proteins in each cell type. The cytosolic Hsp70/110, Hsp60, and Hsp90 initially represent about $1 \%$ of the total proteome in erythroid progenitor stages. Their abundance, however, gradually decreases as the proportion of $\mathrm{Hb}$ increases during erythropoiesis, but much less so than ribosomes and histones. Below, multifaceted functions of the Hsp70 chaperone system at major steps of red blood cell generation. Ability to synthesize proteins is lost in mature erythrocytes. Protein degradation capacity is largely reduced in mature erythrocytes. A selective Hsp70 system seems to drive protein repair in terminally differentiated erythrocytes. PQC: protein quality control. 


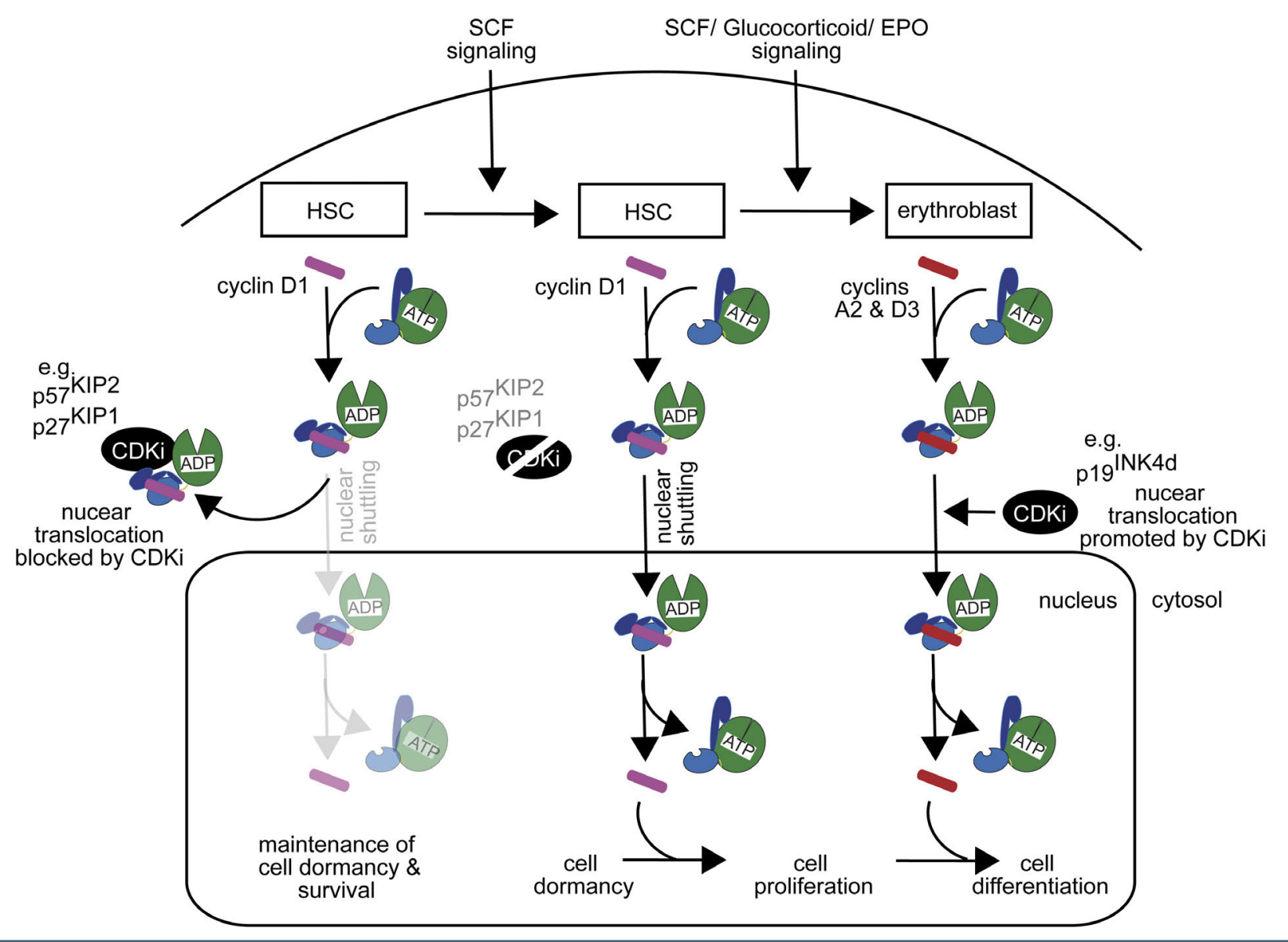

Figure 3. Hsp70 chaperone system modulates the maintenance of dormancy and cell cycle quiescence of stem cell progenitors of erythrocytes. Heat shock protein 70 (Hsp70) binds and shuttles key cyclins that control proliferation and differentiation of erythroid precursor cells. Growth factor regulated cyclin-dependent kinase inhibitors (CDKi) appear to modulate these activities by interacting (directly or indirectly) with Hsp70-cyclin complexes. EPO: erythropoietin; HSC: hematopoietic stem cell; SCF: stem cell factor.

shuttling of cyclins A2 and D3 and co-operate with CDKi to regulate terminal differentiation of erythroblasts. An analysis of erythroblasts obtained from differentiating human cord blood CD34+ cells shows that only three out of the seven CDKi ( $\mathrm{p} 18^{\mathrm{INK} 4 \mathrm{c}}, \mathrm{p} 19^{\mathrm{INK} 4 \mathrm{~d}}$ and $\left.\mathrm{p} 27^{\mathrm{KIPI}}\right)$ are expressed to significant levels during early and late terminal differentiation steps of red blood cells. In contrast to the functions of $\mathrm{p} 57^{\mathrm{KIP} 2}$ and $\mathrm{p} 27^{\mathrm{KP} 1}$ in HSC, $\mathrm{p} 19^{\mathrm{IKK} K d}$ appears to be promoting erythroblast differentiation by facilitating nuclear localization of the stress inducible Hsp70 (HSPA1A) through the activation of the ERK, but not AKT, signal transduction pathway (Figure 3). ${ }^{47}$ p19 $9^{\text {INKKd }}$ may even play a role in the proteostasis-based fitness checkpoints in human erythroblasts (see below). How EPO stimulation leads to the induction and ERK-mediated nuclear translocation of $\mathrm{Hsp} 70$ and the role of CDKi in modulating this process during erythropoiesis remains to be dissected. ${ }^{53,54}$

The mammalian mitochondrial Hsp70 (mortalin/ HSPA9) is also implicated in the proliferation/maintenance of early progenitors of erythrocytes. ${ }^{55}$ HSPA9 cooperates with the inner mitochondrial translocase (TIM) complex to facilitate the translocation of mitochondrial matrix proteins that are essential for mitochondrial function and cell viability. ${ }^{56}$ Therefore, it is conceivable that any depletion of HSPA9 levels leads to increased mitochondrial dysfunction and activation of pro-apoptotic factors that induce hematopoietic progenitor cell death. ${ }^{57}$ Intriguingly, however, compared to progenitors of other lineages of hematopoiesis, a greater reduction of BFU-E progenitors was observed when HSPA9 was knocked down in rodents, suggesting that this Hsp70 paralog possibly plays an additional role(s) in maintaining the erythroid progenitor cell niche. ${ }^{58}$

\section{Hsp70 checks the fitness of erythroblasts at the initiation of erythropoiesis}

The continuous generation of large amounts of red blood cells to traffic $\mathrm{O}_{2} / \mathrm{CO}_{2}$ in vertebrates comes with a heavy energy cost (ATP-wise). By allowing only healthy erythroid progenitors to undergo cell differentiation in part increases the fidelity of this process. In order to select healthy progenitors, two fitness checkpoints seem to have evolved around the Hsp70 chaperone system. In both checkpoints, the Hsp70 chaperone appears to monitor proteostasis deficiencies in EPO stimulated erythroid progenitors that are primed to undergo differentiation. By acting as a sensor of global folding status, Hsp70 is able to gauge the levels of misfolded/aggregated proteins in these cells. The first fitness checkpoint seems to be initiated by momentarily triggering a pro-apoptotic insult during EPO stimulation, which induces mitochondria to undergo transient depolarization. ${ }^{59}$ This prompts the activation of several pro-apoptotic signals including the release of the 
mitochondria localized apoptosis-inducing factor (AIF, AIFM1). ${ }^{59-62}$ The AIF released into the cytosol enters the nucleus via a nuclear localization signal ${ }^{63}$ and initiates caspase-independent chromatin condensation, DNA fragmentation and nuclear shrinkage to fully commit cells to apoptosis. ${ }^{62}$ In healthy erythroblasts, however, Hsp70 chaperone appears to play an important role in neutralizing this pro-apoptotic signaling pathway (Figure 4A). Cytosol-localized Hsp70 (primarily the stress induced HSPA1A) directly interacts with AIF and prevents its translocation into the nucleus..$^{59,63,64}$ On the contrary, in "unhealthy" erythroblasts (e.g., cells experiencing acute oxidative stress), Hsp70 is largely sequestered away by the accumulating misfolded/aggregated proteins. This sequestration prevents the neutralization of AIF signaling, thus leading to fitness checkpoint failure and rapid elimination of unhealthy cells (Figure 4B).

The second checkpoint appears to monitor the capacity of Hsp70 to protect GATA-1 from caspase-3 mediated proteolytic cleavage. EPO stimulation triggers the translocation of HSPA1A from the cytosol to the nucleus. ${ }^{65}$ In healthy erythroblasts, the nuclear translocated HSPA1A binds directly to GATA-1 and prevents the transcription factor from being cleaved, which allows the initiation of erythropoiesis (Figure 4A) ${ }^{65}$ However, if there is a deficiency in Hsp70 levels, activity and/or the chaperone is insufficiently translocated to the nucleus due to the sequestration away by cytosolic protein aggregates, ${ }^{66}$ the unprotected GATA-1 becomes targeted by caspase- 3 (Figure 4B). Reduction in the level of GATA-1 inhibits both the terminal differentiation program and anti-cell death signaling via $\mathrm{Bcl}-\mathrm{xL}^{67}$ that ultimately trigger clearance of unhealthy cells via apoptosis. This fitness checkpoint seems to function throughout the early stages of erythropoiesis. During the latter stage of erythropoiesis, a small heat shock protein (sHSP) named Hsp27 (also know as HSPB1) translocates to the nucleus (triggered by posttranslational modifications) and appears to outcompete Hsp70 from binding to GATA-1, which results in the degradation of the transcription factor. ${ }^{68}$ In a nutshell, i) adequate nuclear and cytosolic levels of "free" Hsp70 chaperones and ii) favorable inputs from both of the proteostasis fitness checkpoints seem to be required to selectively initiate the terminal differentiation of healthy erythroblasts.

\section{Hsp70's role in hemoglobin folding and assembly}

Despite decades of research, the mechanistic understanding of the folding and assembly of $\mathrm{Hb}$ (Figure 1B) remains incomplete. In particular, little is known about how heme moieties are inserted into nascent globin chains during de novo folding. Several chaperones including Hsp70, Hsp90, and AHSP have been recognized to assist in globin folding and assembly in erythrocytes. ${ }^{66,69,70}$ Whether Hsp70 directly assists in Hb biogenesis is still an open question. Early studies have identified a role for Hsp70 in stabilizing $\alpha$-globin and preventing its aggregation during erythropoiesis. ${ }^{6}$ However, this could also result indirectly through the regulation of heme regulated inhibitor of translation (HRI) by Hsp70, which affects $\mathrm{Hb}$ assembly. HRI is the main kinase, which fine-tunes the cellular levels of heme and globin proteins to facilitate efficient $\mathrm{Hb}$ assembly during erythropoiesis..$^{7-73}$ In healthy erythroblasts with sufficient levels of heme, HRI is kept inactive by an autoregulatory mechanism involv- ing complex formation with heme and HSPA8 ${ }^{74,75}$ When cellular heme levels decrease, the inhibition is released and the activated kinase rapidly phosphorylates the eukaryotic translation initiation factor $2 \alpha$ (eIF2 $\alpha$ ). This halts protein synthesis and prevents the overproduction of aggregate-prone globin chains. ${ }^{72,73,76}$ Under proteotoxic stress conditions, HRI is similarly activated to block $\mathrm{Hb}$ production, but now as a result of HSPA8 being sequestrated away by misfolded/aggregated proteins. ${ }^{77}$ This activation appears to be independent of heme levels in erythroblasts. ${ }^{75,77}$ Apart from inhibiting protein translation, HRI initiates an integrated stress response in erythroid precursors by selectively switching-on the transcriptional factor ATF-4 signaling pathway to induce multiple antioxidants that help mitigate oxidative stress. ${ }^{78}$ The triggering of this mechanism can be clearly observed in heat shocked erythroblasts. ${ }^{73,77}$ The activity of HRI is critical for the viability of stressed erythroid progenitors since induction of Hsp70 and other chaperones alone is insufficient to mitigate proteotoxicity in these cells. ${ }^{72,79}$ Additionally, Hsp70 may in part help fold HRI adding another level of complexity to this regulation. ${ }^{75}$ In essence, Hsp70 directly and/or indirectly facilitates the efficient biogenesis of $\mathrm{Hb}$ during erythropoiesis.

\section{Clearance of aberrant proteins by the Hsp70 system during erythropoiesis}

Triggering of ineffective erythropoiesis as a result of increased levels of protein aggregation has been observed in disease conditions such as $\beta$-thalassemia (see section on Hsp70 associated blood disorders). ${ }^{66}$ Interestingly, the induction of Hsp70, but not other major stress chaperones, has been detected in heat shocked erythroblasts.,80 This demonstrates the existence of a somewhat specialized stress response in erythroblasts to perhaps selectively induce Hsp70-based PQC activities. Previous work has shown that rabbit reticulocyte lysates have the capacity to resolve in vitro generated protein aggregates ${ }^{81}$ suggesting that differentiating erythroblasts possess strong protein disaggregation/refolding activity. This activity is most likely generated via the recently discovered Hsp70based protein disaggregases in human cells. ${ }^{82-87}$ These disaggregases could potentially co-operate with cellular protein degradation systems ${ }^{82}$ to rapidly clear aggregated proteins and reduce associated toxicities to facilitate erythopoiesis

\section{Suppression of apoptosis in differentiating erythroblasts by Hsp70}

HSPA1A, which is upregulated in response to proteostasis insults has been demonstrated to block pro-apoptotic pathways that lead to caspase activation in cells. ${ }^{88}$ Erythroblasts appear to rely on the same Hsp70 homolog to prevent premature apoptosis during terminal differentiation. ${ }^{99-91}$ This necessity may partially explain why there is an unusually high level of HSPA1A present even in early erythroid progenitors primed to undergo erythropoiesis (Figure 2B). Additionally, the EPO signaling induced mitochondrial HSPA9 could also inhibit apoptosis in part by suppressing the production of ROS. ${ }^{59}$ These Hsp70 mediated anti-apoptotic signals together with the induction of anti-apoptotic protein Bcl-xL by GATA- $1^{67}$ appears to help prevent erythroblasts from undergoing premature death despite the considerable proteostasis challenges associated with normal erythropoiesis. 
A
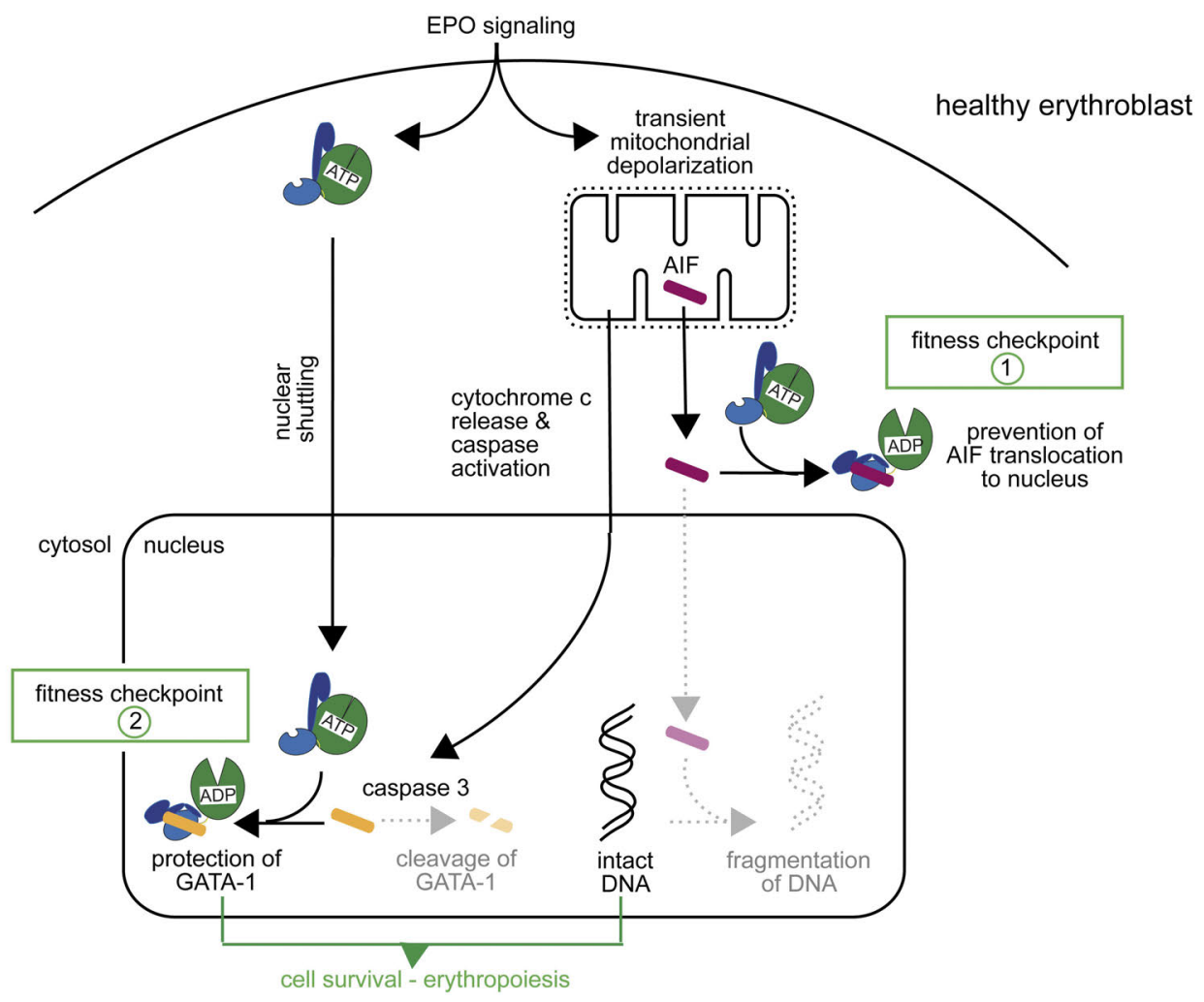

B

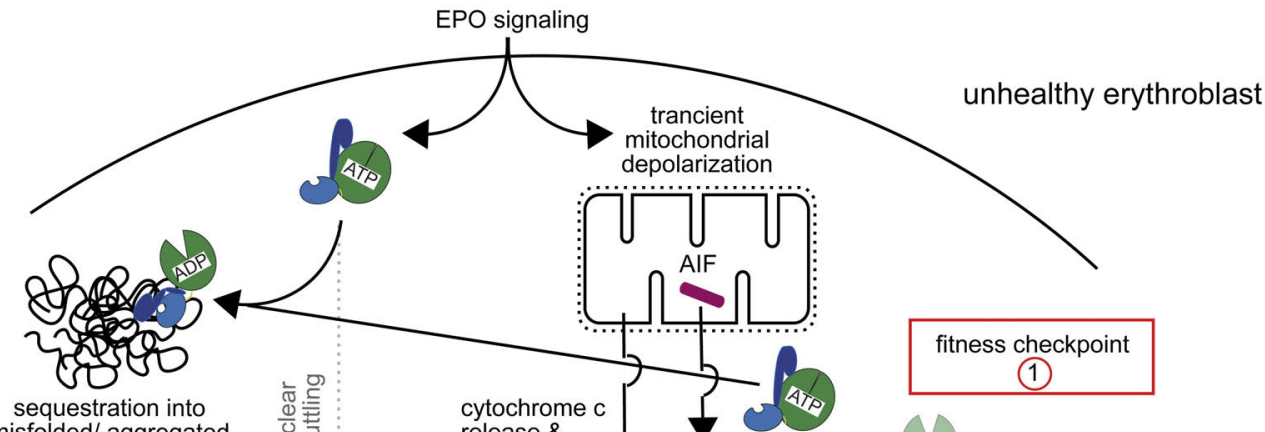
misfolded/ aggregated proteins

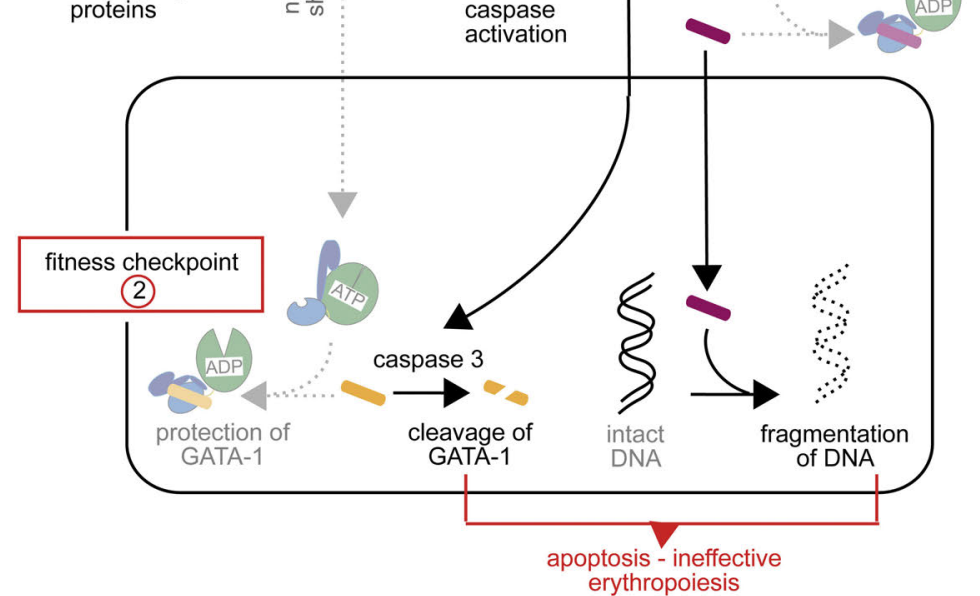

Figure 4. Hsp70 facilitates proper initiation of erythropoiesis. Heat shock protein 70 (Hsp70) is utilized as a stress sensor molecule in two fitness checkpoints to assess proteostasis deficiencies in erythroid progenitors. (A) Checkpoint 1, in essence, "gauges" and tests whether erythroblasts contain sufficient Hsp70 levels in the cytosol to block the nuclear translocation of apoptosis-inducing factor (AIF), a pro-apoptotic factor released from transiently depolarized mitochondria as a result of erythropoietin (EPO) signaling. In unhealthy cells, Hsp70 is sequestered away by protein aggregates and consequently AIF translocates into the nucleus to initiate cell death. Conversely, checkpoint 2 evaluates the ability of Hsp70 to protect GATA-1, the master regulator of erythropoiesis, from caspase 3 cleavage. This too indirectly evaluates the Hsp70 chaperoning capacity in early erythroblasts. Satisfaction from both tests (green) appears to be required to initiate a robust terminal differentiation process. (B) Both fitness checkpoints fail (red) in "unhealthy" cells (e.g., due to increased oxidative stress) containing high levels of misfolded and aggregated proteins that sequester Hsp70. 


\section{Quantitative proteomics highlight vital protein quality control functions related to mature erythrocyte survival}

Mature erythrocytes show a remarkable ability to survive up to 120 days in the circulation ${ }^{6}$ while supporting a plethora of enzymatic reactions required for preserving the cytoskeletal ultrastructure, biomolecule trafficking across membranes and signal transduction (e.g., for maintaining lipid homeostasis ${ }^{92}$ ). Apart from the mechanical stress insults that induce shape changes while navigating through the vasculature, these cells could be subjected to a range of environmental (e.g., chronic hypoxia at high altitudes, hyperosmotic shock, and energy depletion) or chemical (e.g., oxidative stress and high intracellular $\mathrm{Ca}^{2+}$ levels) stresses ${ }^{93}$ that could trigger protein misfolding, damage and/or aggregation. Such conditions could potentially cause eryptosis, a process by which mature erythrocytes undergo apoptosis-like cell death. Unlike other cell types that contain stress-sensing and signaling pathways (e.g., HSF-1 transcription factor-mediated heat shock response) to produce large quantities of new chaperones to help buffer against such proteostasis insults, ${ }^{1}$ mature erythrocytes that lack ribosomes have to utilize already existing PQC elements present in their vestigial proteome to counteract protein misfolding/aggregation.

Advancements in mass spectrometry-based quantitative analytical methods have identified over 2,600 proteins in the vestigial proteome of mature human red blood cells (2-5\% of the progenitor proteome)..$^{94} \mathrm{We}$ performed an enrichment analysis on these proteins or protein classes focusing on function to uncover probable biological processes important for erythrocyte survival. We compared published label-free quantitative proteomics data obtained from complete cytosolic extracts of mature erythrocytes (originating from age-matched, healthy donors), ${ }^{94}$ to human cells (unstressed Jurkat cells) that have not massively accumulated $\mathrm{Hb}$ and carbonic anhydrase, and neither eliminated their transcription and translation machineries, nor lost their endoplasmic reticulum (ER), nuclei and mitochondrial compartments (Figure 5; Online Supplementary Tables S1-4). ${ }^{95}$ As expected, our analysis showed that mature erythrocytes (likely containing $\sim 1 \%$ reticulocytes with ribosomes and ER), ${ }^{94}$ are markedly enriched with $\mathrm{Hb}$, carbonic anhydrase and antioxidant enzymes, such as catalases (Figure 5A). Our analysis also confirmed that mature erythrocytes are severely depleted in DNA polymerases, transcription factors, RNA polymerases, ribosomes, as well as nuclear, ER and mitochondrial proteins compared to Jurkat cells (Figure 5A). On average, the replacement of the proteome with high levels of $\mathrm{Hb}$ and carbonic anhydrases in mature erythrocytes should theoretically reduce the levels of any other given protein by $\sim 280$-fold in abundance compared to Jurkat cells. ${ }^{94,95}$ Thus, it is reasonable to assume that proteins or protein classes that are significantly less depleted than $\sim 280$-fold have been purposefully retained to sustain functions associated with the maintenance and survival of mature erythrocytes. For example, compared to Jurkat cells, the cytoskeletal proteins are 44-fold more abundant than the average non- $\mathrm{Hb}$ protein in erythrocytes thus supporting this view (Figure $5 \mathrm{~A}$ ). We also detected a high level of Hsp60 chaperones that facilitate in the folding and assembly of cytoskeletal proteins. The above observations highlight the critical role of these pro- teins in maintaining cell shape and cytoskeletal integrity of red blood cells.

\section{The role of Hsp70 in terminally differentiated erythrocytes}

Molecular chaperones make up approximately $5 \%$ of the total protein mass of naïve mammalian cells, and up to $10 \%$ in stress-resistant cancer cells, attesting to their central role in maintaining cell viability. The Hsp70-JDP-NEF machinery alone contributes to $\sim 1 \%$ of the total protein mass of most mammalian cells. ${ }^{96}$ Comparatively, the chaperome of mature erythrocytes is $0.28 \%$ of the total protein mass (including Hb) (Online Supplementary Tables S1-2). The erythrocyte Hsp70-JDP-NEF machinery, however, accounts for about a third of the mature erythrocyte chaperome $(0.1 \%$ of the total protein mass; Figure 5; Online Supplementary Table S2). From a proteostasis angle, it is somewhat puzzling as to why this particular chaperone system, which is conventionally thought to function in de novo folding of newly synthesized proteins, ${ }^{97}$ is maintained at relatively high levels in terminally differentiated erythrocytes that lack protein-synthesizing capability.

\section{Up to a certain point, protein repair is less costly than protein replacement}

At the cellular level, life may evade protein decay by maintaining a subtle balance between ATP-fueled protein repair by unfolding chaperones and ATP-fueled protein replacement mediated by controlled degradation of irreversibly damaged proteins followed by transcription, translation, folding and assembly of new functional proteins. Noticeably, in terms of ATP cost, it is energetically cheaper to repair structurally damaged proteins with unfolding chaperones than to degrade them by the proteasome and synthetize replacements at the cost of at least two ATP molecules per peptide bond. ${ }^{37}$

Mature erythrocytes are an extreme case of living cells that completely lack protein replacement mechanisms. This leaves protein repair as the sole mechanism to counteract the time-dependent entropic decay of labile proteins into aggregates and support erythrocyte survival in circulation. Intriguingly, we detected all the components of the Hsp70 chaperone system necessary for protein repair in the vestigial proteome of mature red blood cells. These include the constitutively expressed HSPA8, a selective set of JDP cochaperones (DNAJA and DNAJB) that recognize misfolded/aggregated proteins, and Hsp110-type nucleotide exchange factors that support protein disaggregation/ refolding in human cells (Figure 5C; Online Supplementary Tables S1-2). ${ }^{82-84,86,98,99}$ When combined, the identified Hsp70 chaperone system components were 14-fold more enriched in mature erythrocytes with respect to Jurkat cells (Figure $5 \mathrm{~A}$, red bars). Interestingly, the DNAJA and DNAJB cochaperones showed significant qualitative rearrangements in mature erythrocytes, compared to Jurkat cells (Figures $5 \mathrm{~B}$ and $\mathrm{C})$. The JDP composition shifted from a DNAJA:DNAJB ratio of $\sim 2: 1$ in Jurkat cells (Figure $5 B$ ) to a DNAJB class-dominated ratio (approximately DNAJA:DNAJB = 1:9) in erythrocytes (Figure 5C). Hsp110 cochaperones were also re-arranged in erythrocytes; HYOU1 (the ER-resident HSP110) and HSPH3 (APG-1, cytosolic) were considerably depleted, while the proportion of HSPH2 (APG-2, cytosolic) was only slightly decreased from $~ 9 \%$ (of the Hsp70/110 chaperome) in Jurkat cells to $\sim 7 \%$ in total erythrocyte cytosol (Figures $5 \mathrm{~B}$ and $\mathrm{C}$ ). In 
A

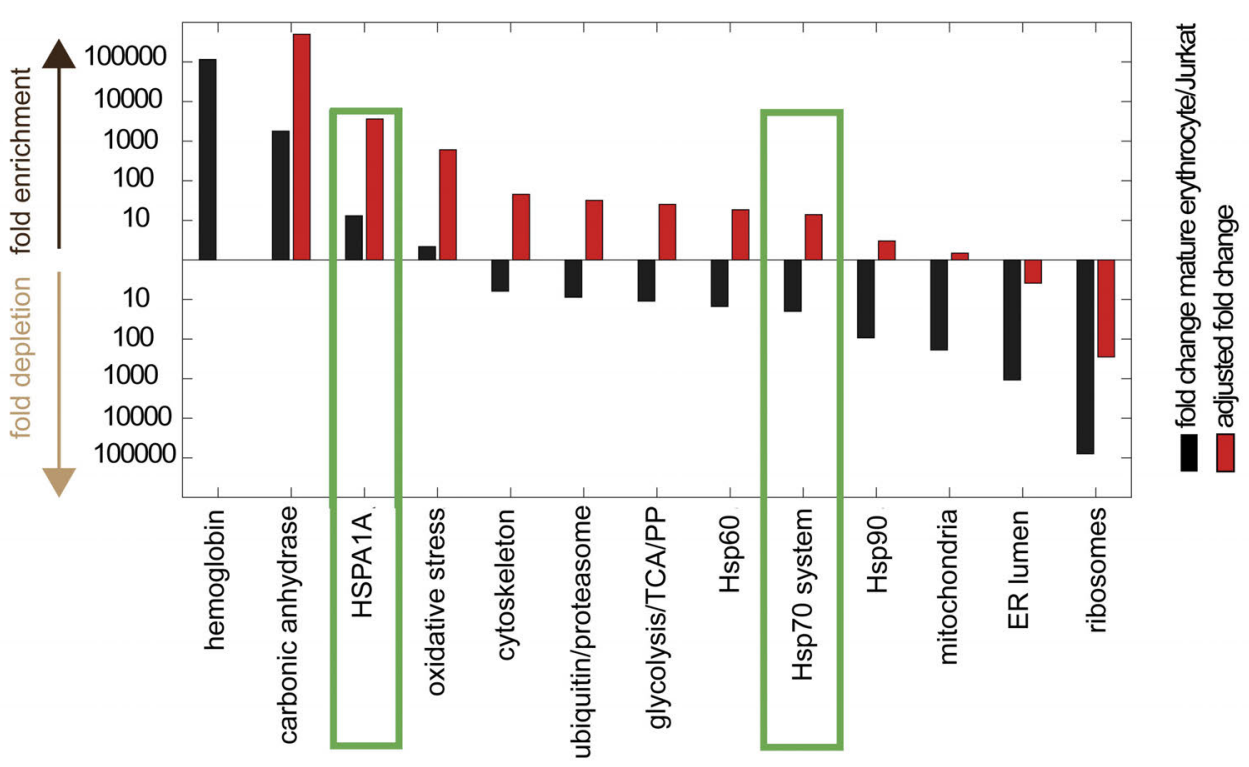

B

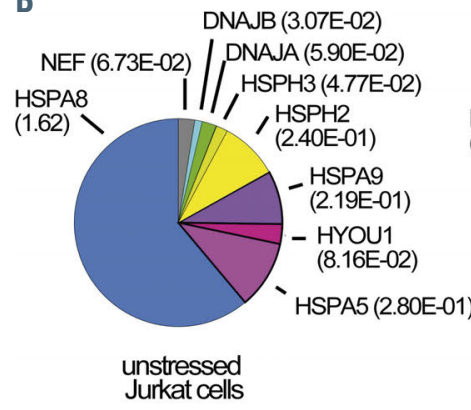

C

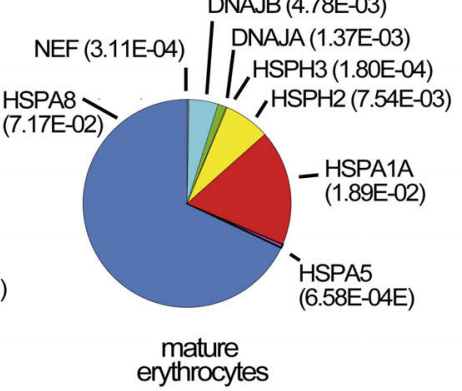

G

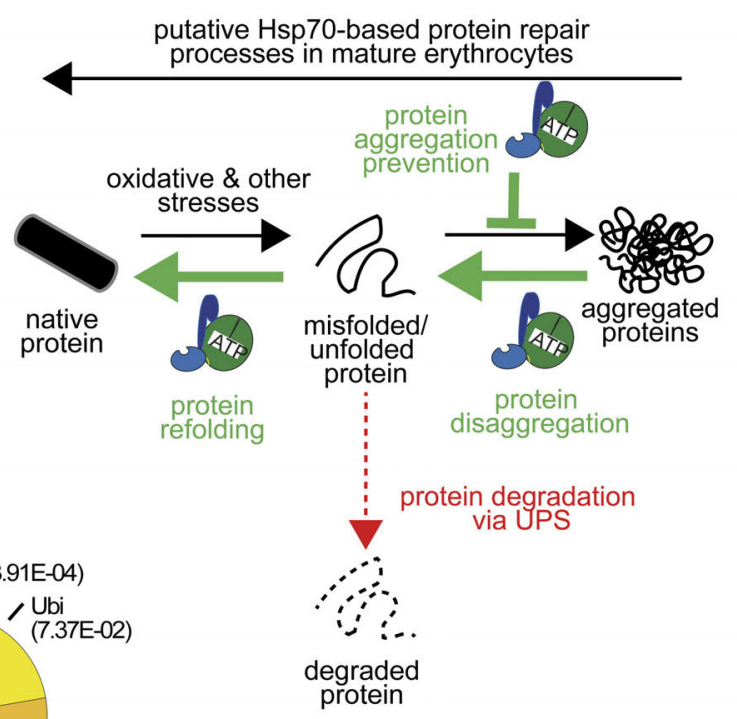

D

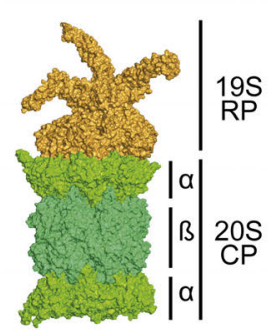

E

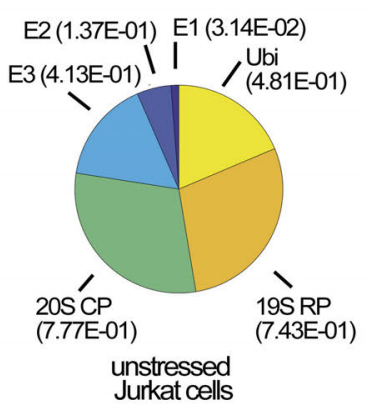

$\mathrm{F}$

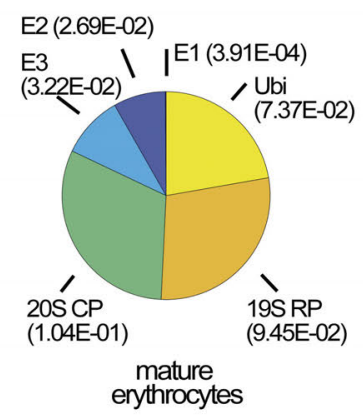


essence, quantitative proteomic analysis revealed a strong bias towards maintaining a set of fully operational Hsp70 machineries conceivably functioning in specific protein repair activities important for the survival of mature erythrocytes (Figure 5G). We also noticed a clear enrichment of the stress-induced form of Hsp70, HSPA1A (13-fold more enriched than in Jurkat cells, and $>3,500$ times more abundant than the average non-Hb protein) in mature erythrocytes (Figures 5A and C). Analysis of the levels of this chaperone during red blood cell formation indicated that the HSPA1A is present 83-fold higher even in erythroblast progenitors compared to unstressed Jurkat cells, and then only decreases approximately 10-fold during terminal differentiation (Figure 2B; Online Supplementary Figure S1). The high levels of HSPA1A perhaps largely facilitate the blocking of erythroblasts from undergoing premature apoptosis during differentiation. ${ }^{89-91}$ However, in mature erythrocytes, HSPA1A may serve a different purpose where it could also form chaperone machines that primarily solubilize and repair misfolded/aggregated proteins. Functional studies are now required to deconvolute from the alternative, whereby some of the more abundant chaperones such as the Hsp70 linger in mature erythrocytes simply because of an incomplete proteome reduction.

We also noticed the retention of a fully functional UPS, perhaps more fine-tuned to the needs of mature erythrocyte maintenance (Figures 5A, D to F; Online Supplementary Tables S3-4). Although UPS proteins are 10fold less abundant in erythrocytes than in Jurkat cells, they are 32-times less depleted than the average non- $\mathrm{Hb}$ protein (Figure $5 \mathrm{~A}$ ). A basal protein degradation system is likely needed to prevent the cytotoxic accumulation of terminally-damaged proteins in these cells (Figure 5G). As expected, the E2-E3 hybrid enzyme UBE2O was present in mature erythrocytes perhaps to more selectively target unpaired/damaged $\alpha$-globin chains. ${ }^{27}$ We also, however, observed a considerable enrichment of Cullin-RING E3 ubiquitin ligase family members. It is somewhat puzzling as to why such E3 ubiquitin ligases are retained in postmitotic terminally differentiated erythrocytes, given that their functions are mainly associated with gene transcription, cell cycle and development. ${ }^{100}$ In contrast, we observed the absence of E3 ubiquitin ligases that target misfolded proteins for degradation, such as members of the UBR family ${ }^{101-103}$ and STUB1/CHIP, which directly binds and ubiquitinate Hsp70 substrates ${ }^{104}$ (Online Supplementary Tables S3-4). This implies that protein degradation is considerably regulated and clearance of misfolded proteins might be kept to a minimal to further promote protein repair over proteolysis in mature erythrocytes. Importantly, we also detected an enrichment of metabolic enzymes required to support glycolysis and the pentose phosphate cycle. These catabolic and metabolic pathways are vital for importing and breaking down glucose to produce ATP. The ATP generated from an active glycolysis reaction could supply the energy needed to support (i) chaperone-based protein repair (ii) UPSmediated protein degradation and (iii) active ion pumps required for maintaining the steep ion gradients across plasma membrane in erythrocytes. Taking everything into account, it is intriguing to speculate that the retained Hsp70 chaperone system together with the UPS is cogged towards primarily repairing proteins in mature red blood cells. This is of particular interest given the wide array of hematological diseases associated with $\mathrm{Hb}$ aggregation.

\section{Hsp70 associated blood disorders}

The Hsp70 chaperone has been implicated in the pathophysiology of several prominent blood disorders in humans. Below we describe how this chaperone system may act as an important modifier which influences both the severity and progression of hematological disorders such as $\beta$-thalassemia, sickle cell disease, myelodysplastic syndromes, polycythemia vera and Diamond Blackfan anemia. Importantly, the ineffective erythropoiesis observed in these disorders is intimately linked to several key functions of the Hsp70 chaperone system in red blood cell differentiation.

\section{Mislocalization of Hsp70 drives ineffective erythropoiesis in $\beta$-thalassemia}

$\beta$-thalassemia is an autosomal recessive disease with three clinical phenotypes: $\beta$-thalassemia major (severe anemia), intermedia (mild to moderate anemia) and minor (clinically asymptomatic, patients act as "carriers" of the disease). One hallmark of this disease is the premature apoptosis of differentiating erythroblasts in the bone marrow and the rapid destruction of circulating erythrocytes by the reticuloendothelial system. $\beta$-thalassemia arises from a series of point mutations and deletions that reduce or prevent the production of functional $\beta$-globin. The decrease in $\beta$-globin levels correlates with the severity of the condition. ${ }^{105}$ Apart from the mutation driven $\beta$ globin dosage effect, it was also found that the co-inheritance of the genetic variants of the globin genes has a modifying effect on the severity of the disease, ${ }^{105,106}$ which could be partly attributed to protein misfolding and aggregation. At a mechanistic level, the decrease in $\beta$-globin levels leads to increased aggregation of unpaired $\alpha$ globin chains, ${ }^{107}$ which triggers acute oxidative stress in erythroblasts leading to premature apoptosis. ${ }^{108}$

The Hsp70 chaperone system plays a pivotal role in the pathogenesis of $\beta$-thalassemia. During erythropoiesis, the stress-induced form of Hsp70 (HSPA1A) translocates in to the nucleus to protect GATA- 1 from caspase- 3 cleavage and initiate terminal differentiation (Figure 4) ${ }^{65}$ However in $\beta$-thalassemia, this translocation step is largely impeded as a result of Hsp70 being sequestered into cytosolic aggregates formed by excess unpaired $\alpha$-globin chains. ${ }^{66}$ This ultimately results in GATA-1 cleavage, which triggers ineffective erythropoiesis leading to anemia. In parallel, sequestration of cytosolic Hsp70 by protein aggregates could also i) compromise the overall chaperoning capacity of early erythroblasts and ii) affect protein synthesis due to HRI activation. ${ }^{76}$ Although, a reduction in protein synthesis may temporally help prevent further accumulation of aggregate-prone proteins, such as $\alpha$-globin chains, ${ }^{76}$ it may also considerably affect the overall $\mathrm{Hb}$ biogenesis in these already compromised red blood cells. ${ }^{77}$ This mechanism may partly contribute to microcytosis that causes mild anemia in $\beta$-thalassemia minor.

A recent breakthrough study showed an unexpected suppression of the disease phenotype in a mouse model of $\beta$-thalassemia intermedia when UBE2O, which helps clear unpaired $\alpha$-globin chains, was knocked out $\left(\mathrm{Hb}^{\mathrm{th} 3 /+}\right.$ Ube $\left.20^{-/}\right) .{ }^{27}$ The mitigation of anemia in these animals resulted from an increase in erythrocyte levels. Intriguingly, the erythrocytes generated in $\mathrm{Hbb}^{\text {th } 3 /+} \mathrm{Ube20^{-1 }}$ animals were relatively healthier to those that were produced in $\mathrm{Hbb}^{\text {th3/+ }}$ genetic background. The $\mathrm{Hbb}^{\mathrm{th} 3 /+} \mathrm{Ube} 2 \mathrm{o}^{-1-}$ 
erythrocytes also showed a dramatic decrease in the levels of aggregated $\mathrm{Hb}$. The authors speculated that the observed decrease in $\alpha$-globin aggregation resulted from an increase in eIF2 $\alpha$ phosphorylation, which reduced the production of globin chains (approximately $20 \%$ and $40 \%$ reduction in $\alpha$ - and $\beta$-globin, respectively, compared to control animals). ${ }^{27}$ However, the steady-state $\alpha$-globin: $\beta$-globin ratio in the soluble protein fraction, in which the aggregate prone $\alpha$-globin was still largely in excess,

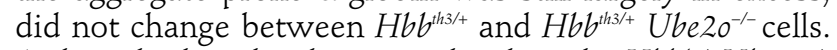
A closer look at the chaperone levels in the $\mathrm{Hbb}^{\text {this }} \mathrm{Ube}^{-1} \mathrm{o}^{-1-}$ erythrocytes suggests an alternative explanation. The study shows that the deletion of UBE2O resulted in elevated levels of AHSP. Similarly, the Hsp70 and Hsp90 chaperone systems were also induced in these cells. ${ }^{27}$ Such chaperone inductions could boost the protein repair capacity consequently leading to the observed decrease in $\alpha$-globin precipitation in $\mathrm{Hb}^{\mathrm{th} \mathrm{b}^{3 /+}} \mathrm{Ube} 2 \mathrm{o}^{-/}$erythroblasts despite defects in degrading excess $\alpha$-globin. The moderate decrease in $\alpha$-globin synthesis may also help reduce the burden on PQC machineries contributing to the remarkable rescue of erythroblasts in the $\mathrm{Hbb}^{\text {tisl }} \mathrm{Ube} 2 \mathrm{O}^{-/-}$ animals. Based on the findings from our proteomics data analysis (Figure 5), we speculate that the degradation of a certain amount of damaged proteins, despite the inability to replace them, might be tolerated and perhaps advantageous for the long-term survival of mature erythrocytes under healthy conditions. However, in unhealthy erythroblasts (e.g., early $\beta$-thalassemic erythroblasts ${ }^{108}$ ), a strong induction of the UPS due to stress could generate an aberrant $\mathrm{PQC}$ condition where even foldable conformers of proteins might be partitioned towards rapid degradation. ${ }^{101}$ Such a condition could disrupt the fine balance between protein repair and clearance in these cells (Figure 5G). A careful study of the potential misregulation of PQC pathways in erythroblasts is required to fully comprehend the underlining mechanism of pathology in $\beta$ thalassemia. It is tempting to speculate that even slight increases in the levels of certain Hsp70 machineries at pre- or very early stages of erythropoiesis may favorably tilt the folding equilibrium of globin chains and minimize the formation of cytotoxic $\mathrm{Hb}$ aggregates. Such chaperone manipulations at clinical level could result in reducing the symptoms of $\beta$-thalassemia.

\section{Hsp70, a key modulator of inflammation in sickle cell disease?}

Sickle cell disease (SCD) is another autosomal recessive genetic disorder associated with chronic anemia. SCD results from massive cyclic-polymerization of a structurally aberrant variant of adult $\mathrm{Hb} \mathrm{S}(\mathrm{HbS})$ under hypoxic conditions. The resulting $\mathrm{HbS}$ fibers deform mature erythrocytes into rigid "sickle" shaped cells that aggregate and readily undergo premature destruction in the vasculature. Whether these conditionally formed reversible protein fibers (distinct from amyloid-type fibers formed e.g., in neurodegenerative disorders) trigger any proteostasis insult involving the Hsp70 system is unknown. The Hsp70 chaperone, however, may play an important role in activating the inflammatory response in SCD..$^{109,10}$ Stressed cells have been observed to secrete Hsp70 into the extracellular matrix. ${ }^{109}$ Immune cells generate specific peptides from secreted Hsp70 that act as key mediators of stress-induced inflammation. ${ }^{11-113} \mathrm{~A}$ considerable buildup of circulating Hsp70 levels have been detected in patients with SCD ${ }^{109}$ suggesting that there is an active secretion of Hsp70 by blood cells. Under hypoxic conditions, sickled erythrocytes show differential recruitment of the stress-inducible HSPA1A to the cell membrane,,$^{114}$ possibly representing an early step in this secretion process. Interestingly, a similar observation was recently noted in $\beta$-thalassemia intermedia. ${ }^{115}$ Together, the observations suggest that the high levels of extracellularly circulating Hsp70 may serve as an important immune modulator that trigger inflammation in these hemoglobinopathies, leading to increased red blood cell destruction by macrophages.

\section{Haploinsufficiency of Hsp70 associated with myelodysplastic syndromes}

Myelodysplastic syndromes (MDS) are a heterogeneous, but closely related group of hematopoietic malignancies characterized by ineffective erythropoiesis leading to peripheral blood cytopenias. ${ }^{116}$ The mitochondria localized HSPA9 is strongly implicated as a protein that contributes to MDS. The HSPA9 locus (5q31.2) is frequently deleted in patients with MDS, leading to a haploinsufficiency of this chaperone. ${ }^{117}$ Recent work showed that mutating HSPA9 causes an MDS-like phenotype in zebrafish $^{118}$ and a knockdown in rodents results in considerable delay in erythroid progenitor maturation. ${ }^{58}$ HSPA9 is implicated in the pathogenesis of MDS at two levels. First, the haploinsufficiency of the chaperone may contribute to the phenotype as a result of altered mitochondrial import and refolding of heme-synthesis enzymes required for heme biogenesis during erythropoiesis. ${ }^{55}$ Second, a decrease in HSPA9 could activate p53, a nuclear transcription factor, resulting in cell cycle arrest and premature apoptosis of hematopoietic progenitor cells. ${ }^{57}$

Further, a recent study demonstrated that defects in EPO induced nuclear translocation of Hsp70 in erythroblasts could also be an important driver of these disorders. ${ }^{119}$ The ineffective erythropoiesis observed in MDS could largely be reversed by protecting GATA-1 from caspase 3 cleavage using an Hsp70 variant (lacking the nuclear export signal) that accumulates in the nucleus. ${ }^{119}$ Protein aggregates containing aberrant p53 (as in cancer cells) $)^{120,121}$ have been detected in erythroblasts in some forms of MDS. ${ }^{122}$ These aggregates could conceivably sequester Hsp70, thus triggering ineffective erythropoiesis in a similar mechanism to that in $\beta$-thalassemia. Alternatively, defective EPO signaling or/and other mechanisms affecting nuclear transportation of proteins, could lead to the Hsp70 trafficking defect observed in MDS. ${ }^{119}$

\section{Hsp70 is a modifier of polycythemia vera}

Polycythemia vera is a hyperproliferative disorder characterized by increased synthesis of red blood cells resulting in hyperviscosity of whole-blood. Recent proteomic studies on polycythemia vera have shown that increased levels of Hsp70 along with Hsp90 stabilize JAK2 kinase. This triggers a prolonged aberrant activation of the kinase, which results in massive proliferation of erythroid progenitors and abnormal stimulation of erythropoiesis. ${ }^{123-126}$ Inhibition of either Hsp70 or Hsp90 has been demonstrated to promote the apoptosis of the abnormally proliferating erythroid progenitors and is currently being investigated as a potential therapeutic approach to delay the progression of this disease in humans. ${ }^{123,126}$ 


\section{Haploinsufficiency of Hsp70 modulates Diamond Blackfan anemia}

Diamond Blackfan anemia (DBA) is a rare congenital bone marrow failure syndrome resulting from ineffective erythropoiesis. ${ }^{127}$ In this disease, erythroid differentiation arrests between BFU-E and CFU-E stages.128 More than $70 \%$ of the cases of DBA occur due to haploinsufficiency of genes that encode for the small and large ribosomal subunit proteins. ${ }^{129-131}$ The defective ribosome biogenesis triggers ineffective erythropoiesis in part due to the decreased production of GATA-1. ${ }^{132}$ This is further promoted by imbalances in globin chain and heme synthesis leading to $\alpha$-globin aggregation and induction of oxidative stress in erythroblasts ${ }^{133}$ similar to $\beta$-thalassemia. Additionally, in DBA associated with RPL11, but not RPL19 haploinsufficiency, the Hsp70 chaperone is considerably degraded in the erythroblasts via the UPS. ${ }^{133,134}$ The reason for the rapid degradation of $\mathrm{Hsp70}$ in some permutations of this disorder remains unclear. Recent work shows that aberrations in chromatin organization resulting from low levels of the global chromatin organizer SATB1 prevents the induction of Hsp70 in early erythroblasts in DBA. ${ }^{135}$ Together, these observations suggest that the differential Hsp70 expression and degradation rates may have considerable effect on red blood cell viability, differentiation and $\mathrm{Hb}$ biogenesis, thus partly explaining the variability in the observed phenotypes of DBA. Remarkably, the restoration of Hsp70 levels in affected erythroblasts inhibits premature apoptosis and substantially restores erythropoiesis in $\mathrm{DBA},{ }^{133,134}$ thus providing an important therapeutic avenue for the treatment of this blood disorder.

\section{A predicted pathological role for Hsp70 in congenital sideroblastic anemias}

Congenital sideroblastic anemias (CSA) are inherited rare blood disorders characterized by erythroblasts displaying ring sideroblasts formed by the pathological depositions of iron in mitochondria. Patients with CSA show a significant reduction in the regeneration of erythrocytes leading to anemic conditions. The ineffective erythropoiesis in CSA is caused by defects in iron-sulfur cluster biogenesis essential for a broad range of cellular functions. Patients with CSA show mutations in genes directly (e.g., GLRX5, $A B C B 7)^{136,137}$ and indirectly (e.g., HSPA 9) $)^{138,139}$ associated with this biosynthetic pathway. It is important to note that the ring sideroblasts are uncommon in MDS cases that are also associated with HSPA9 deletion mutations (see above), but whether this is due to an epistatic suppression by another modifier remains to be investigated. ${ }^{138}$ As in CSA, HSPA9 may also play an indirect role in some forms of dyserythropoietic anemias that are also characterized by pathological iron-loading defects and ineffective erythropoiesis ${ }^{140}$ and warrants further investigation.

\section{Age-associated anemia}

Aging is attributed to a decline in hematopoiesis with high incidents of anemia. ${ }^{141}$ The viability and self-renewal of HSC/early erythroid progenitors depend on maintaining robust PQC activity and high levels of Hsp70. ${ }^{142,143,144}$ These abilities decline in stem cells during aging. ${ }^{145,146}$ As a consequence, Hsp70-mediated functions such as maintenance of erythroid dormancy, cell cycle quiescence and cell cycle entry may breakdown leading to an age-related exhaustion of HSC.

\section{Concluding remarks}

Recent findings have considerably broadened our understanding of the multifaceted roles of Hsp70 in erythrocyte differentiation and how deficiencies in its activity modify several blood disorders in humans. The tuning of the Hsp70 chaperone system to cater to different POC needs during erythropoiesis sheds extremely valuable insight on cell repair and viability and provides a conceptual framework for investigating chaperone-based therapeutic avenues for a wide spectrum of blood disorders.

\section{Disclosures}

No conflicts of interest to disclose.

\section{Contributions}

NBN conceptualized the work. BF, PG and NBN analyzed published proteomics data. YM, BF, SJ, PG and NBN wrote and edited the manuscript.

\section{Acknowledgements}

We thank Andrew Perkins (Australian Center for Blood Diseases) David Ron (Cambridge Institute for Medical Research) for critically reading the manuscript.

\section{Funding}

This work was supported by a special Recruitment Grant from the Monash University Faculty of Medicine Nursing and Health Sciences with funding from the State Government of Victoria and the Australian Government to NBN.

\section{References}

1. Labbadia J, Morimoto RI. The biology of proteostasis in aging and disease. Annu Rev Biochem. 2015;84:435-464

2. Vonk WI, Rainbolt TK, Dolan PT, Webb AE, Brunet A, Frydman J. Differentiation drives widespread rewiring of the neural stem cell chaperone network. Mol Cell. 2020;78:329345.

3. Gautier E-F, Ducamp S, Leduc M, et al. Comprehensive proteomic analysis of human erythropoiesis. Cell Rep. 2016;16(5):1470-1484

4. Banerji SS, Theodorakis N, Morimoto RI. Heat shock-induced translational control of HSP70 and globin synthesis in chicken retic- ulocytes. Mol Cell Biol. 1984;4(11):24372448.

5. Gautier EF, Leduc M, Cochet S, et al. Absolute proteome quantification of highly purified populations of circulating reticulocytes and mature erythrocytes. Blood Adv. 2018;2(20): 2646-2657.

6 . Hall JE. Red blood cells, anemia and polycythemia. Guyton and Hall Textbook of Medical Physiology 13 ed. Philadelphia: Elsevier Health Sciences; 2015. Chapter 33:445-454.

7. Higgins JM. Red blood cell population dynamics. Clin Lab Med. 2015;35(1):43-57.

8. Li H, Natarajan A, Ezike J, et al. Single cell resolution of glucocorticoid effects on erythroid progenitor cells. Blood. 2018;132
(Suppl 1):S751.

9. Peslak SA, Wenger J, Bemis JC, et al. EPOmediated expansion of late-stage erythroid progenitors in the bone marrow initiates recovery from sublethal radiation stress. Blood. 2012;120(12):2501-2511

10. Wierenga AT, Vellenga E, Schuringa JJ. Down-regulation of GATA1 uncouples STAT5-induced erythroid differentiation from stem/progenitor cell proliferation. Blood. 2010;115(22):4367-4376.

11. Chiba T, Ikawa Y, Todokoro K. GATA-1 transactivates erythropoietin receptor gene, and erythropoietin receptor-mediated signals enhance GATA-1 gene expression Nucleic Acids Res. 1991;19(14):3843-3848.

12. Bain BJ, Bates I, Laffan MA. Reference 
ranges and normal values. In: Lewis SM, editor. Dacie and Lewis Practical Haematology. 12 ed. China: Elsevier 2017:2-17

13. Roux-Dalvai F, Gonzalez de Peredo A, Simo C, et al. Extensive analysis of the cytoplasmic proteome of human erythrocytes using the peptide ligand library technology and advanced mass spectrometry. Mol Cell Protiomics. 2008;7(11):2254-2269.

14. Goloubinoff P, Sassi AS, Fauvet B, Barducci A, De Los Rios P. Chaperones convert the energy from ATP into the nonequilibrium stabilization of native proteins. Nat Chem Biol. 2018;14(4):388-395.

15. Stefani M. Protein misfolding and aggregation: new examples in medicine and biology of the dark side of the protein world. Biochim Biophys Acta. 2004;1739(1):5-25.

16. Voon HPJ, Vadolas J. Controlling $\alpha$-globin: a review of $\boldsymbol{\alpha}$-globin expression and its impact on $\beta$-thalassemia. Haematologica. 2008;93 (12):1868-1876

17. Bank A. Hemoglobin synthesis in $\beta$-thalassemia: the properties of the free $\alpha$-chains. J Clin Invest. 1968:47(4):860-866.

18. Fibach E, Dana M. Oxidative stress in betathalassemia. Mol Diagn Ther. 2019;23(2): 245-261.

19. Feng L, Gell DA, Zhou S, et al. Molecular mechanism of AHSP-mediated stabilization of $\alpha$-hemoglobin. Cell. 2004;119(5):629-640.

20. Mollan TL, Khandros E, Weiss MJ, Olson JS. Kinetics of $\alpha$-globin binding to $\alpha$-hemoglobin stabilizing protein (AHSP) indicate preferential stabilization of hemichrome folding intermediate. J Biol Chem. 2012;287(14): 11338-11350.

21. Weiss MJ, Zhou S, Feng L, et al. Role of alpha hemoglobin stabilizing protein in normal erythropoiesis and $\beta$-thalassemia. Ann NY Acad Sci. 2005;1054(1):103-117.

22. Barrett KE, Barman SM, Brooks H, Yuan J. Ganong's review of medical physiology. 26 ed. New York: McGraw-Hill Medical; 2019:543-551.

23. Drummond DA, Wilke CO. The evolutionary consequences of erroneous protein synthesis. Nat Rev Genet. 2009;10(10):715

24. Camaschella C, Hoffbrand AV, Hershko C. Iron metabolism, iron deficiency and disorders of haem synthesis. Postgrad Haematol. 2015:21-39.

25. Kruszewski M. Labile iron pool: the main determinant of cellular response to oxidative stress. Mutat Res. 2003:531(1-2):81-92.

26. Yanagitani K, Juszkiewicz S, Hegde RS. UBE2O is a quality control factor for orphans of multiprotein complexes. Science. 2017;357(6350):472-475

27. Nguyen AT, Prado MA, Schmidt PJ, et al. UBE2O remodels the proteome during terminal erythroid differentiation. Science. 2017;357(6350):471.

28. Pilla E, Schneider K, Bertolotti A. Coping with protein quality control failure. Annu Rev Cell Dev Biol. 2017;33:439-465.

29. Mizuno S. Temperature sensitivity of protein synthesis initiation: inactivation of a ribosomal factor by an inhibitor formed at elevated temperatures. Arch Biochem Biophys. 1977;179(1):289-301.

30. Rosenzweig R, Nillegoda NB, Mayer MP, Bukau B. The Hsp70 chaperone network. Nat Rev Mol Cell Biol. 2019;11:665-680.

31. Kampinga HH, Craig EA. The HSP70 chaperone machinery: J-proteins as drivers of functional specificity. Nat Rev Mol Cell Biol. 2010;11(8):579-592.

32. Kityk R, Kopp J, Mayer MP. Molecular mechanism of J-domain-triggered ATP hydrolysis by Hsp70 chaperones. Mol Cell. 2018;69(2):227-237.
33. Cyr DM. Swapping nucleotides, tuning Hsp70. Cell. 2008;133(6):945-947.

34. Finka A, Mattoo RU, Goloubinoff $P$ Experimental milestones in the discovery of molecular chaperones as polypeptide unfolding enzymes. Annu Rev Biochem. 2016:85:715-742.

35. De Los Rios P, Barducci A. Hsp70 chaperones are non-equilibrium machines that achieve ultra-affinity by energy consumption. Elife. 2014;3:e02218

36. Schrödinger E. What is life? The physical aspect of the living cell. Cambridge University Press; 1944.

37. Sharma SK, De los Rios P, Christen P, Lustig A, Goloubinoff P. The kinetic parameters and energy cost of the Hsp70 chaperone as a polypeptide unfoldase. Nat Chem Biol 2010;6(12):914-920.

38. Trinklein ND, Chen WC, Kingston RE, Myers RM. Transcriptional regulation and binding of heat shock factor 1 and heat shock factor 2 to 32 human heat shock genes during thermal stress and differentiation. Cell Stress Chaperon. 2004:9(1):21.

39. Saretzki G, Armstrong L, Leake A, Lako M, von Zglinicki T. Stress defense in murine embryonic stem cells is superior to that of various differentiated murine cells. Stem Cells. 2004;22(6):962-971

40. Matsumoto A, Takeishi S, Kanie T, et al. p57 is required for quiescence and maintenance of adult hematopoietic stem cells. Cell Stem Cell. 2011;9(3):262-271.

41. Zou P, Yoshihara H, Hosokawa K, et al. p57Kip2 and p27Kip1 cooperate to maintain hematopoietic stem cell quiescence through interactions with Hsc70. Cell Stem Cell. 2011;9(3):247-261.

42. Tesio M, Trumpp A. Breaking the cell cycle of HSCs by p 57 and friends. Cell Stem Cell. 2011;9(3):187-192.

43. Böcking T, Aguet F, Harrison SC, Kirchhausen T. Single-molecule analysis of a molecular disassemblase reveals the mechanism of Hsc70-driven clathrin uncoating. Nat Struct Mol. 2011;18(3):295

44. Chakraborty A, Mukherjee S, Chattopadhyay R, Roy S, Chakrabarti S. Conformational adaptation in the $\mathrm{E}$. coli sigma 32 protein in response to heat shock. J Phys Chem. 2014;118(18):4793-4802.

45. Marcinowski M, Höller M, Feige MJ, Baerend D, Lamb DC, Buchner J. Substrate discrimination of the chaperone $\mathrm{BiP}$ by autonomous and cochaperone-regulated conformational transitions. Nat Struct Cell Biol. 2011;18(2): 150

46. Zhang J, Socolovsky M, Gross AW, Lodish HF. Role of Ras signaling in erythroid differentiation of mouse fetal liver cells: functional analysis by a flow cytometry-based novel culture system. Blood. 2003;102(12):39383946

47. Han X, Zhang J, Peng Y, et al. Unexpected role for p19INK4d in posttranscriptional regulation of GATA1 and modulation of human terminal erythropoiesis. Blood. 2017;129(2): 226-237.

48. Sterrenberg JN, Blatch GL, Edkins AL. Human DNAJ in cancer and stem cells. Cancer Lett. 2011;312(2):129-142.

49. Zhang Y, Yang Z, Cao Y, et al. The Hsp40 family chaperone protein DnaJB6 enhances Schlafen1 nuclear localization which is critical for promotion of cell-cycle arrest in $\mathrm{T}$ cells. Biochem J. 2008;413(2):239-250.

50. Watson ED, Mattar P, Schuurmans C, Cross IC. Neural stem cell self-renewal requires the Mrj co-chaperone. Dev Dynam. 2009;238(10):2564-2574.

51.Ludwig LS, Cho H, Wakabayashi A, et al
Genome-wide association study follow-up identifies cyclin $\mathrm{A} 2$ as a regulator of the transition through cytokinesis during terminal erythropoiesis. Am J Hematol. 2015;90(5): 386-391.

52. Sankaran VG, Ludwig LS, Sicinska E, et al. Cyclin D3 coordinates the cell cycle during differentiation to regulate erythrocyte size and number. Genes Dev. 2012;26(18):20752087.

53. Arai A, Kanda E, Miura O. Rac is activated by erythropoietin or interleukin-3 and is involved in activation of the Erk signaling pathway. Oncogene. 2002;21(17):2641.

54. Song H, Kim W, Kim S-H, Kim K-T. VRK3mediated nuclear localization of HSP70 prevents glutamate excitotoxicity-induced apoptosis and $A \beta$ accumulation via enhancement of ERK phosphatase VHR activity. Sci Rep. 2016;6:38452.

55. Shan Y, Cortopassi G. Mitochondrial Hspa9/Mortalin regulates erythroid differentiation via iron-sulfur cluster assembly. Mitochondrion. 2016;26:94-103

56. Yamamoto H, Momose T, Yatsukawa Y-i, et al. Identification of a novel member of yeast mitochondrial Hsp70-associated motor and chaperone proteins that facilitates protein translocation across the inner membrane. FEBS Lett. 2005;579(2):507-511.

57. Liu T, Krysiak K, Shirai CL, et al. Knockdown of HSPA9 induces TP53-dependent apoptosis in human hematopoietic progenitor cells. PLoS One. 2017;12(2): e0170470

58. Chen TH-P, Kambal A, Krysiak K, et al Knockdown of Hspa9, a del (5q31. 2) gene, results in a decrease in hematopoietic progenitors in mice. Blood. 2011;117(5):1530 1539

59. Weiss MJ, dos Santos CO. Chaperoning erythropoiesis. Blood. 2009;113(10):2136-2144.

60. Zermati Y, Garrido C, Amsellem S, et al. Caspase activation is required for terminal erythroid differentiation. J Exp Med. 2001;193(2):247-254

61. Kolbus A, Pilat S, Husak Z, et al. Raf-1 antagonizes erythroid differentiation by restraining caspase activation. J Exp Med. 2002;196(10):1347-1353.

62. Cande C, Vahsen N, Garrido C, Kroemer G Apoptosis-inducing factor (AIF): caspaseindependent after all. Cell Death Differ. 2004;11(6):591.

63. Gurbuxani S, Schmitt E, Cande C, et al. Heat shock protein 70 binding inhibits the nuclear import of apoptosis-inducing factor. Oncogene. 2003;22(43):6669.

64. Lui JC-K, Kong S-K. Heat shock protein 70 inhibits the nuclear import of apoptosisinducing factor to avoid DNA fragmentation in TF-1 cells during erythropoiesis. FEBS Lett. 2007;581(1):109-117.

65. Ribeil JA, Zermati Y, Vandekerckhove J, et al. Hsp70 regulates erythropoiesis by preventing caspase-3-mediated cleavage of GATA-1. Nature. 2007;445(7123):102-105.

66. Arlet JB, Ribeil JA, Guillem F, et al. HSP70 sequestration by free alpha-globin promotes ineffective erythropoiesis in beta-thalassaemia. Nature. 2014;514(7521):242-246.

67. Gregory T, Yu C, Ma A, Orkin SH, Blobel GA, Weiss MI. GATA-1 and erythropoietin cooperate to promote erythroid cell survival by regulating bcl-xL expression. Blood. 1999:94(1):87-96.

68. de Thonel A, Vandekerckhove J, Lanneau D, et al. HSP27 controls GATA-1 protein level during erythroid cell differentiation. Blood. 2010;116(1):85-96.

69. Ghosh A, Garee G, Sweeny EA, Nakamura Y, Stuehr DJ. Hsp90 chaperones hemoglobin 


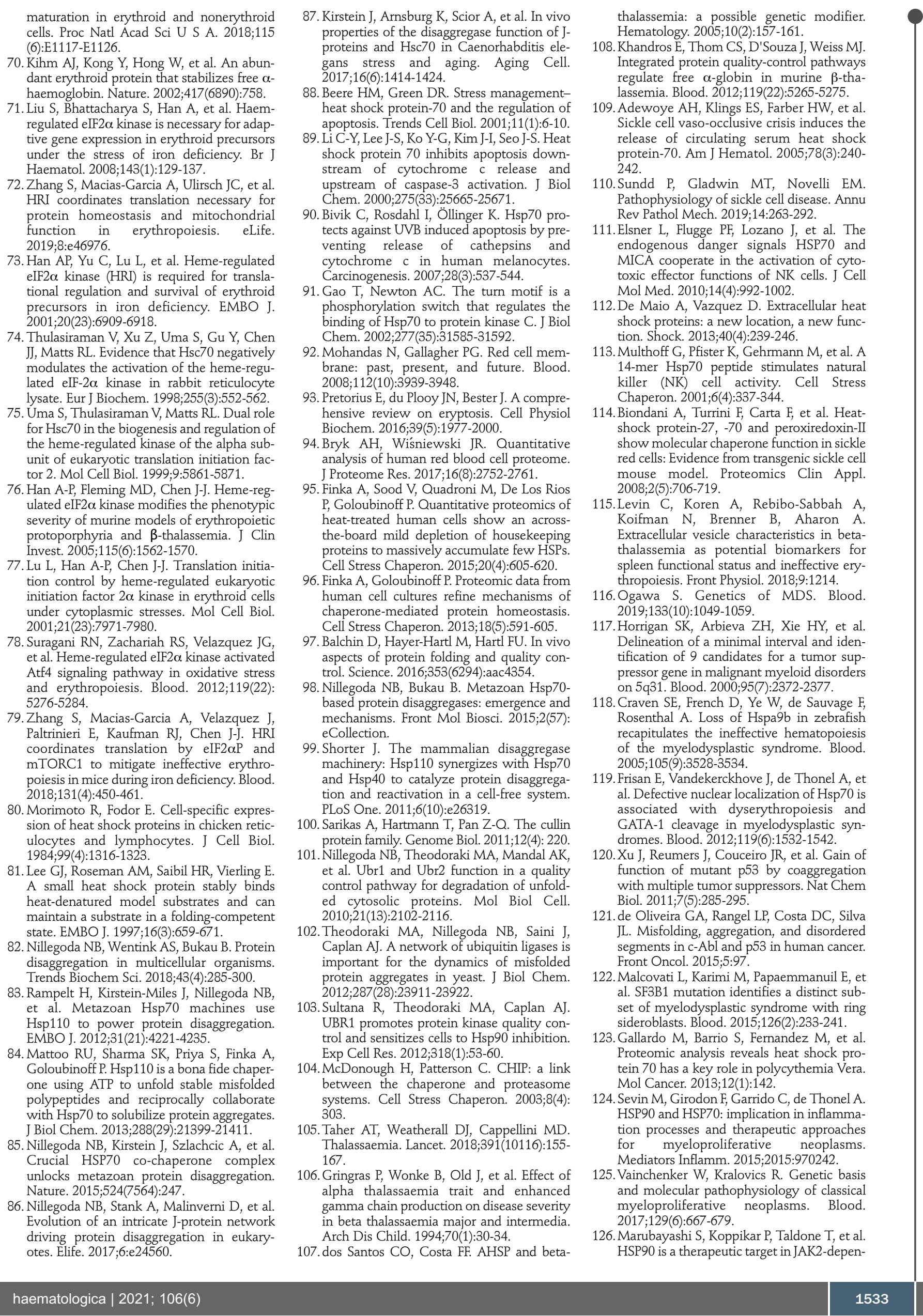


dent myeloproliferative neoplasms in mice and humans. J Clin Invest. 2010;120(10): 3578-3593.

127. Nathan DG, Clarke BJ, Hillman DG, Alter $\mathrm{BP}$, Housman DE. Erythroid precursors in congenital hypoplastic (Diamond-Blackfan) anemia. J Clin Invest. 1978;61(2):489-498.

128. Ohene-Abuakwa Y, Orfali KA, Marius C, Ball SE. Two-phase culture in Diamond Blackfan anemia: localization of erythroid defect. Blood. 2005;105(2):838-846.

129. Choesmel V, Fribourg S, Aguissa-Touré A-H, et al. Mutation of ribosomal protein RPS24 in Diamond-Blackfan anemia results in a ribosome biogenesis disorder. Hum Mol Genet. 2008;17(9):1253-1263.

130. Farrar JE, Nater M, Caywood E, et al. Abnormalities of the large ribosomal subunit protein, Rpl35a, in Diamond-Blackfan anemia. Blood. 2008;112(5):1582-1592.

131. Quarello P, Garelli E, Brusco A, et al. High frequency of ribosomal protein gene deletions in Italian Diamond-Blackfan anemia patients detected by multiplex ligationdependent probe amplification assay. Haematologica. 2012;97(12):1813-1817.

132. Ludwig LS, Gazda HT, Eng JC, et al. Altered translation of GATA1 in Diamond-Blackfan anemia. Nat Med. 2014;20(7):748-753.

133. Rio S, Gastou M, Karboul N, et al. Regulation of globin-heme balance in Diamond-Blackfan anemia by HSP70/GATA1. Blood. 2019;133(12):1358-1370.

134. Gastou M, Rio S, Dussiot M, et al. The severe phenotype of Diamond-Blackfan anemia is modulated by heat shock protein 70. Blood Adv. 2017:1(22):1959-1976.

135. Wilkes MC, Takasaki K, Youn M, Chae H-
D, Narla A, Sakamoto KM Chromatin Organization By SATB1 Regulates HSP70 Induction in Early Erythropoiesis and Lost in Diamond Blackfan Anemia. Blood. 2018;132(Suppl 1):S2591.

136. Allikmets R, Raskind WH, Hutchinson A, Schueck ND, Dean M, Koeller DM Mutation of a putative mitochondrial iron transporter gene ( $\mathrm{ABC7}$ ) in X-linked sideroblastic anemia and ataxia (XLSA/A). Hum Mol Genet. 1999;8(5):743-749.

137. Liu G, Guo S, Anderson GJ, Camaschella C, Han B, Nie G. Heterozygous missense mutations in the GLRX5 gene cause sideroblastic anemia in a Chinese patient. Blood. 2014;124(17):2750-2751.

138. Schmitz-Abe K, Ciesielski SJ, Schmidt PJ, et al. Congenital sideroblastic anemia due to mutations in the mitochondrial HSP70 homologue HSPA9. Blood. 2015;126(25): 2734-2738.

139. Furuyama K, Kaneko K. Iron metabolism in erythroid cells and patients with congenital sideroblastic anemia. Int $\mathrm{J}$ Hematol. 2018;107(1):44-54

140. Lefêvre C, Bondu S, Le Goff S, Kosmider O, Fontenay M. Dyserythropoiesis of myelodysplastic syndromes. Curr Opin Hematol. 2017;24(3):191-197.

141. Gazit R, Weissman IL, Rossi DJ. Hematopoietic stem cells and the aging hematopoietic system. Semin Hematol. 2008;45(4):218-224.

142.Pang Q, Keeble W, Christianson TA, Faulkner GR, Bagby GC. FANCC interacts with Hsp70 to protect hematopoietic cells from IFN- $\gamma /$ TNF- $\alpha$-mediated cytotoxicity. EMBO J. 2001;20(16):4478-4489.
143. Mortensen M, Soilleux EJ, Diordievic G, et al. The autophagy protein Atg7 is essential for hematopoietic stem cell maintenance. J Exp Med. 2011;208(3):455-467.

144. De Franceschi L, Bertoldi M, De Falco L, et al. Oxidative stress modulates heme synthesis and induces peroxiredoxin- 2 as a novel cytoprotective response in $\beta$-thalassemic erythropoiesis. Haematologica. 2011;96(11): 1595-1604.

145.Higuchi-Sanabria R, Frankino PA, Paul III JW, Tronnes SU, Dillin A. A futile battle? Protein quality control and the stress of aging. Dev Cell. 2018;44(2):139-163.

146. Vilchez D, Saez I, Dillin A. The role of protein clearance mechanisms in organismal ageing and age-related diseases. Nat Commun. 2014;5:5659.

147. Zhuravleva A, Clerico EM, Gierasch LM. An interdomain energetic tug-of-war creates the allosterically active state in Hsp70 molecular chaperones. Cell. 2012;151(6):1296-1307.

148. Rudiger S, Germeroth L, SchneiderMergener J, Bukau B. Substrate specificity of the DnaK chaperone determined by screening cellulose-bound peptide libraries. EMBO J. 1997;16(7):1501-1507.

149. Pesciotta EN, Lam H-S, Kossenkov A, et al. In-depth, label-free analysis of the erythrocyte cytoplasmic proteome in diamond blackfan anemia identifies a unique inflammatory signature. PLoS One. 2015;10(10): e0140036.

150. Cox J, Mann M. MaxQuant enables high peptide identification rates, individualized ppb-range mass accuracies and proteomewide protein quantification. Nat Biotechnol. 2008;26(12):1367. 